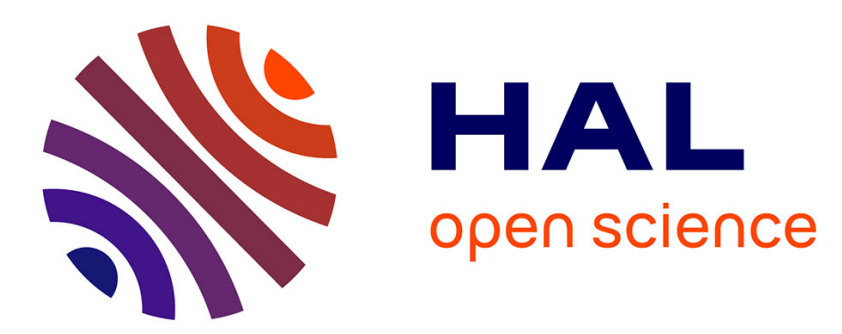

\title{
Pointwise estimates for Laplace equation. Applications to the free boundary of the obstacle problem with Dini coefficients
}

\author{
Régis Monneau
}

\section{- To cite this version:}

Régis Monneau. Pointwise estimates for Laplace equation. Applications to the free boundary of the obstacle problem with Dini coefficients. 2007. hal-00144787

\author{
HAL Id: hal-00144787 \\ https://hal.science/hal-00144787
}

Preprint submitted on 4 May 2007

HAL is a multi-disciplinary open access archive for the deposit and dissemination of scientific research documents, whether they are published or not. The documents may come from teaching and research institutions in France or abroad, or from public or private research centers.
L'archive ouverte pluridisciplinaire HAL, est destinée au dépôt et à la diffusion de documents scientifiques de niveau recherche, publiés ou non, émanant des établissements d'enseignement et de recherche français ou étrangers, des laboratoires publics ou privés. 


\title{
Pointwise estimates for Laplace equation. Applications to the free boundary of the obstacle problem with Dini coefficients
}

\author{
R. Monneau*
}

May 4, 2007

\begin{abstract}
In this paper we are interested in pointwise regularity of solutions to elliptic equations. In a first result, we prove that if the modulus of mean oscillation of $\Delta u$ at the origin is Dini (in $L^{p}$ average), then the origin is somehow a Lebesgue point of continuity (still in $L^{p}$ average) for the second derivatives $D^{2} u$. We extend this pointwise regularity result to the obtacle problem for the Laplace equation with Dini right hand side at the origin. Under these assumptions, we prove that the solution to the obstacle problem has a kind of Taylor expansion up to the order 2 (in the $L^{p}$ average). Moreover we get a quatitative estimate of the error in this Taylor expansion for regular points of the free boundary. In the case where the right hand side is moreover double Dini at the origin, we also get a quatitative estimate of the error for singular points of the free boundary.

Our method of proof is based on some decay estimates obtained by contradiction, using blow-up arguments and Liouville Theorems. In the case of singular points, our method uses moreover a refined monotonicity formula.
\end{abstract}

AMS Classification: 35R35.

Keywords: Obstacle problem, Laplace equation, Dini regularity, free boundary, singular set, pointwise regularity.

\section{Introduction}

\subsection{The Laplace equation}

In this paper, we are interested in the pointwise regularity properties of solutions to elliptic problems. We first consider the solutions to the following Laplace equation

$$
\left\{\begin{array}{l}
\Delta u=f \quad \text { on } \quad B_{1} \\
f \in L^{p}\left(B_{1}\right) \text { and } f(0)=0
\end{array}\right.
$$

*CERMICS, Ecole nationale des Ponts et Chaussées, 6 et 8 avenue Blaise Pascal, Cité Descartes, Champssur-Marne, 77455 Marne-la-Vallée Cedex 2 
where in $\mathbb{R}^{n}$, we denote by $B_{r}=B_{r}(0)$ the open ball of radius $r$ and of center the origin 0 . Here $p \in(1,+\infty)$, and we only assume that 0 is a Lebesgue point of $f$, in order to define $f(0)$ when it is necessary.

It is well-known that, if $f$ is Hölder continuous on the ball $B_{1}$, then this is also true for the second derivatives of the solution $u$ (see for instance Gilbarg, Trudinger [25] for a classical proof of this result based on the potential theory).

Let us introduce the following modulus of continuity of $f$ on the ball $B_{1}$ :

$$
\bar{\sigma}(r)=\sup _{|x-y| \leq r, x, y \in B_{1}}|f(x)-f(y)|
$$

Definition 1.1 (Dini modulus of continuity / double Dini)

A modulus of continuity $\bar{\sigma}$ is said Dini if it satisfies the following integral condition:

$$
\int_{0}^{1} \frac{\bar{\sigma}(r)}{r} d r<+\infty
$$

It is said double Dini if

$$
\int_{0}^{1} \frac{d r}{r}\left(\int_{0}^{r} \frac{d s}{s} \bar{\sigma}(s)\right)<+\infty
$$

It is also well-known that if $\bar{\sigma}$ is Dini, then the second derivatives of the solution $u$ are continuous in any ball striclty contained in $B_{1}$, with a modulus of continuity which is proportional to $\int_{0}^{r} \bar{\sigma}(s) d s / s+r \int_{r}^{1} \bar{\sigma}(s) d s / s^{2}$. For proofs based on potential theory, see Hartman, Wintner [27], Matiichuk, Eidel'man [31], Burch [4], and for a proof based on Dini-Campanato spaces and explicit approximations of the solution by polynomials, see for instance Kovats [29]. For similar results for the regularity of solutions of elliptic systems in divergence form based on the harmonic approximation lemma, see Duzaar, Gastel [20], Duzaar, Gastel, Mingione [21], and also Wolf [39] with a different approach.

The previous results where obtained assuming a modulus of continuity in an open set. Here we change the point of view, and only want to consider pointwise modulus of mean oscillation. For any $p \in(1,+\infty)$, let us introduce the following modulus of mean oscillation (in $L^{p}$ average) of the function $f$ at the origin:

$$
\tilde{\sigma}_{p}(\rho)=\sup _{r \in(0, \rho]} \inf _{c \in \mathbb{R}}\left(\frac{1}{\left|B_{r}\right|} \int_{B_{r}}|f(x)-c|^{p}\right)^{\frac{1}{p}}
$$

Let us denote by $\tilde{\mathcal{P}}_{2}$ the set of polynomials of degree less or equal to 2 , and let us set

$$
\tilde{M}(u, \rho)=\sup _{r \in(0, \rho]}\left(\inf _{P \in \tilde{\mathcal{P}}_{2}}\left(\frac{1}{r^{n+2 p}} \int_{B_{r}}|u-P|^{p}\right)^{\frac{1}{p}}\right)
$$

Theorem 1.2 (Pointwise BMO estimates for the Laplace equation)

Let $p \in(1,+\infty)$ given. Then there exist $\alpha \in(0,1]$ and constants $C>0, r_{0} \in(0,1)$, such 
that, given a function $u \in L^{p}\left(B_{1}\right)$ satisfying (1.1) with a modulus of mean oscillation $\tilde{\sigma}_{p}$ defined in (1.5), we have

$$
\forall r \in(0,1), \quad \tilde{M}(u, r) \leq C\left\{\left(\int_{B_{1}}|u|^{p}\right)^{\frac{1}{p}}+\left(\int_{B_{1}}|f|^{p}\right)^{\frac{1}{p}}+\tilde{\sigma}_{p}(1)\right\}
$$

Moreover if $\tilde{\sigma}_{p}$ is Dini, then $\tilde{M}(u, \cdot)$ is Dini, and there exists a harmonic polynomial $P_{0}$ of degree less or equal to 2 , such that for every $r \in\left(0, r_{0}\right)$ :

$$
\left(\frac{1}{\left|B_{r}\right|} \int_{B_{r}}\left|\frac{u(x)-P_{0}(x)}{r^{2}}\right|^{p}\right)^{\frac{1}{p}} \leq C\left(\tilde{M}_{0} r^{\alpha}+\int_{0}^{r} \frac{\tilde{\sigma}_{p}(s)}{s} d s+r^{\alpha} \int_{r}^{1} \frac{\tilde{\sigma}_{p}(s)}{s^{1+\alpha}} d s\right)
$$

and

$$
P_{0}(x)=a+b \cdot x+\frac{1}{2} t x \cdot c \cdot x
$$

with

$$
|a|+|b|+|c| \leq C \tilde{M}_{0} \quad \text { and } \quad \tilde{M}_{0}=\int_{0}^{1} \frac{\tilde{\sigma}_{p}(s)}{s} d s+\left(\int_{B_{1}}|u|^{p}\right)^{\frac{1}{p}}+\left(\int_{B_{1}}|f|^{p}\right)^{\frac{1}{p}}
$$

Here $b \cdot x$ denotes the scalar product between the vectors $b$ and $x$.

Remark 1.3 In other words, Theorem 1.2 shows in particular that we have somehow a Lebesgue point of continuity of the second derivatives $D^{2} u$ (in the $L^{p}$ average) if $\Delta u$ has a Dini modulus of mean oscillation (in the $L^{p}$ average) at the same point.

Remark 1.4 A straightforward corollary of Theorem 1.2 gives in particular that the second derivatives $D^{2} u$ are Hölder continuous in an open set $\Omega$, if $\Delta u$ is Hölder continuous in $\Omega$.

Theorem 1.2 gives a kind of Taylor expansion up to the second order with a quantitative estimate of the rest in the $L^{p}$ norm. This notion of continuity of derivatives seems quite natural and is related to the notion of approximate derivatives (for $p=1$, see section 2.9 of Federer [24], for $p=n$ see Caffarelli [10], see also Campanato spaces [18], the generalized Campanato-John-Nirenberg spaces in [12], the $t_{2}^{p}$ class of Calderon, Zygmund [17], or the notion of generalized derivatives in Diederich [19]).

We would like to emphasis that the result of Theorem 1.2 is completely pointwise, which does not seem so usual in the literature (see for instance the article of Simon [36] obtaining Schauder estimates by a scaling argument joint to compactness). Even if part of this result is somehow contained in a proof of Kovats [29] in the special case $p=+\infty$ (see the proof of his Lemma 2.1), our method of proof is completely different. Here we do not use an explicit construction of approximate polynomials, but on the contrary prove the result by contradiction, using a blow-up argument. The consequence is that we do not recover the best exponent $\alpha=1$. Nevertheless, we think that our method of proof is quite flexible, and present below new consequences for the obstacle problem. 


\subsection{The model obstacle problem}

In this article we are in particular interested in the regularity of the free boundary for solutions to obstacle problems. The model problem is the following. We consider bounded functions $u$, satisfying on the unit ball $B_{1}$, for $p \in(\max (n / 2,1),+\infty)$

$$
\left\{\begin{array}{l|l}
\Delta u=f(x) \cdot 1_{\{u>0\}} & \text { on } B_{1} \\
u \geq 0 & \\
u, f \in L^{p}\left(B_{1}\right) \quad \text { and } \quad f(0)=1 \\
0 \in \partial\{u>0\}
\end{array}\right.
$$

where $1_{\{u>0\}}$ is the characteristic function of the set $\{u>0\}$ which is equal to 1 if $u>0$ and 0 if $u=0$. From classical elliptic estimates joint to Sobolev imbbedings with our assumption $p>n / 2$, every solution $u$ is in particular continuous, which allows us to consider the boundary of the open set $\{u>0\}$. Here $\partial\{u>0\}$ is called the free boundary. We assume that 0 is a Lebesgue point of $f$ in order to define $f(0)$.

Let us introduce the following pointwise modulus of continuity (in $L^{p}$ average) of the function $f$ at the origin:

$$
\sigma_{p}(\rho)=\sup _{r \in(0, \rho]}\left(\frac{1}{\left|B_{r}\right|} \int_{B_{r}}|f(x)-f(0)|^{p}\right)^{\frac{1}{p}}
$$

We have the following general regularity result.

\section{Proposition 1.5 (Quadratic growth)}

Let $p \in(\max (n / 2,1),+\infty)$. Then there exists constants $C>0$ such that if $u$ is a solution of (1.8) with $\sigma_{p}$ bounded given by (1.9), then

$$
\forall x \in B_{1 / 2}, \quad 0 \leq u(x) \leq C_{1}|x|^{2} \quad \text { with } \quad C_{1}=C\left\{\left(\int_{B_{1}}|u|^{p}\right)^{\frac{1}{p}}+\sigma_{p}(1)+1\right\}
$$

When we assume furthermore that $p \geq 2 n /(n+1)$, it is interesting to present the following preliminary result which distinguishes if 0 is a degenerate, regular or a singular point of the free boundary.

Theorem 1.6 (Definition of degenerate/regular/singular points by the monotonicity formula)

Given a solution $u$ of (1.8), we define for $r \in(0,1)$

$$
\Phi(r)=\frac{1}{r^{n+2}} \int_{B_{r}} \frac{1}{2}|\nabla u|^{2}+u-\frac{1}{r^{n+3}} \int_{\partial B_{r}} u^{2}
$$

for some $p \in(\max (n / 2,1),+\infty)$ with $p \geq 2 n /(n+1)$. If the modulus of continuity $\sigma_{p}$ defined in (1.9) is Dini, then $\Phi$ has a limit at $r=0$, that we denote by $\Phi\left(0^{+}\right)$. Moreover there exists a constant $\alpha=\alpha(n)>0$, such that either 
i) $\Phi\left(0^{+}\right)=0$ and then the point 0 is called a degenerate point, or

ii) $\Phi\left(0^{+}\right)=\alpha$ and then the point 0 is called a regular point, or

iii) $\Phi\left(0^{+}\right)=2 \alpha$ and then the point 0 is called a singular point.

Moreover in the special case where $f \equiv 1$, the function $\Phi$ is nondecreasing in $r$.

Remark 1.7 Under the present assumptions, it is possible to build examples (see Section 5) with degenerate points. On the contrary, if we assume moreover that $f \geq \delta_{0}>0$, then it is classical that 0 can not be a degenerate point (see Caffarelli ["7], Blank [5]).

Let us recall that this result is originally du to Weiss [38] for $f \equiv 1$ (see also Monneau [32] for a version for some Dini modulus of continuity, and Petrosyan, Shahgholian [34] for a similar monotonicity formula for double Dini modulus of continuity, but for obstacle problems with no sign condition on the solution).

Let us introduce the following quantity (which is finite by Proposition 1.5)

$$
M_{r e g}(u, \rho)=\sup _{r \in(0, \rho]}\left(\inf _{P \in \mathcal{P}_{r e g}}\left(\frac{1}{r^{n+2 p}} \int_{B_{r}}|u-P|^{p}\right)^{\frac{1}{p}}\right)
$$

where

$$
\mathcal{P}_{\text {reg }}=\left\{P, \quad \exists \nu \in \mathbf{S}^{n-1}, \quad P(x)=\frac{1}{2} \max (0, x \cdot \nu)^{2}\right\}
$$

Our main results are the following three statements in the regular case and in the singular case.

Theorem 1.8 (Modulus of continuity at a regular point of the free boundary)

Let $p \in(\max (n / 2,1),+\infty)$. There exists $\alpha \in(0,1]$ and constants $C>0, M_{0}, r_{0} \in(0,1)$ such that, given a function u satisfying (1.8), we have the following property.

If the modulus of continuity $\sigma_{p}$ defined in (1.9) is assumed Dini, and if

$$
M_{r e g}\left(u, r_{0}\right) \leq M_{0}
$$

then there exists $P_{0} \in \mathcal{P}_{\text {reg }}$ such that for every $r \in\left(0, r_{0}\right)$ :

$$
\left(\frac{1}{\left|B_{r}\right|} \int_{B_{r}}\left|\frac{u(x)-P_{0}(x)}{r^{2}}\right|^{p}\right)^{\frac{1}{p}} \leq C\left(M_{r e g}\left(u, r_{0}\right) r^{\alpha}+\int_{0}^{r} \frac{\sigma_{p}(s)}{s} d s+r^{\alpha} \int_{r}^{1} \frac{\sigma_{p}(s)}{s^{1+\alpha}} d s\right)
$$

Remark 1.9 With the same methods of proof, it would be possible to get a similar estimate for any $p \in(1,+\infty)$, but under the stronger assumption that the coefficient of the right hand side of the equation is bounded from above and from below, i.e. $0<\delta_{0} \leq f \leq 1 / \delta_{0}$.

\section{Theorem 1.10 (Uniqueness of the blow-up limit at singular points)}

Let $p \in(\max (n / 2,1),+\infty)$ and $p \geq 2 n /(n+1)$ with $p \geq 2$. If the modulus of continuity $\sigma_{p}$ defined in (1.9) is assumed Dini, then there exists a non-negative polynomial $P_{0}$ homogeneous of degree 2 satisfying $\Delta P_{0}=1$, such that

$$
\left(\frac{1}{\left|B_{r}\right|} \int_{B_{r}}\left|\frac{u(x)-P_{0}(x)}{r^{2}}\right|^{2}\right)^{\frac{1}{2}} \longrightarrow 0 \quad \text { as } \quad r \longrightarrow 0
$$


Let us define the set

$$
\mathcal{P}_{\text {sing }}=\left\{P, \quad \exists Q \in \mathbb{R}_{\text {sym }}^{n \times n}, \quad P=\frac{1}{2} t x \cdot Q \cdot x, \quad \text { trace }(Q)=1, \quad Q \geq 0\right\}
$$

We also define

$$
M_{\text {sing }}(u, \rho)=\sup _{r \in(0, \rho]}\left(\inf _{P \in \mathcal{P}_{\text {sing }}}\left(\frac{1}{r^{n+4}} \int_{B_{r}}|u-P|^{2}\right)^{\frac{1}{2}}\right)
$$

Then we have

Theorem 1.11 (Modulus of continuity at a singular point of the free boundary) Let $p \in(n / 2,+\infty)$ with $p \geq 2 n /(n+1)$ and $p \geq 2$. There exists $\alpha \in(0,1]$ and constants $C>0, M_{0}, r_{0} \in(0,1)$ such that, given a function $u$ satisfying (1.8), we have the following property.

If the modulus of continuity $\sigma_{p}$ defined in (1.9) is assumed double Dini, and if

$$
M_{\text {sing }}\left(u, r_{0}\right) \leq M_{0}
$$

then there exists $P_{0} \in \mathcal{P}_{\text {sing }}$ such that for every $r \in\left(0, r_{0}\right)$ :

$$
\left(\frac{1}{\left|B_{r}\right|} \int_{B_{r}}\left|\frac{u(x)-P_{0}(x)}{r^{2}}\right|^{2}\right)^{\frac{1}{2}} \leq C\left(M_{\text {sing }}\left(u, r_{0}\right) r^{\alpha}+\int_{0}^{r} \frac{\Sigma_{p}(s)}{s} d s+r^{\alpha} \int_{r}^{1} \frac{\Sigma_{p}(s)}{s^{1+\alpha}} d s\right)
$$

with

$$
\Sigma_{p}(s)=\sigma_{p}(s)+\int_{0}^{s} d t \frac{\sigma_{p}(t)}{t}
$$

Remark 1.12 In particular the boundary $\partial\left\{P_{0}>0\right\}$ can be interpretated as the tangent $j$ dimensional subspace to the free boundary at the origin 0 . Here $j=n-1$ for regular points, and $j=\operatorname{dim} \operatorname{Ker} Q$ for singular points.

Remark 1.13 Theorem 1.11 stays true if we replace $\Sigma_{p}(s)$ by

$$
\tilde{\Sigma}_{p}(s)=\sigma_{p}(s)+\left(\int_{0}^{s} d t \frac{\sigma_{p}^{2}(t)}{t}\right)^{\frac{1}{2}}
$$

assuming only that $\tilde{\Sigma}_{p}$ is Dini. This is a sharper result, because we always have $\tilde{\Sigma}_{p} \leq C \Sigma_{p}$ and for instance for $\sigma_{p}(s)=|\ln s|^{-\frac{3}{2}}$, we have that $\tilde{\Sigma}_{p}$ is Dini while $\Sigma_{p}$ is not.

Let us emphasis again that these results are pointwise, and seem the first pointwise results for the obstacle problem, up to our knowledge. The regularity of the free boundary can be easily deduced from these theorems (see Theorem 8.3).

Concerning regular points, let us mention slightly more precise results on the regularity of the free boundary when the modulus of continuity is not only controled at the origin 0 , but controled at any points (see Blank [5] for sharp results). See the previous works of Caffarelli [6, 7, 8, 9], Weiss [38], and Caffarelli, Karp, Shahgholian [13] for Lipschitz coefficients, and also the work of Caffarelli, Kinderlehrer [14] for some related estimates on the modulus 
of continuity of the solution or of its gradient. Let us mention that very recently, similar regularity results have been obtained in Petrosyan, Shahgholian [34] for the regular points of the free boundary, for an obstacle problem with no sign assumption on the solution. These results are obtained under geometric and energetic conditions and the assumption that $\int_{0}^{1} d r \frac{\bar{\sigma}(r) \ln \frac{1}{r}}{r}$ is finite, which can easily be seen to be equivalent to the double Dini assumption. See also Lee, Shahgholian [30] for regularity results for fully nonlinear obstacle problems.

Concerning singular points, the first result of regularity has been proved by Caffarelli [8] using the monotonicity formula of Alt, Caffarelli, Friedman [1], and this result has been generalized for Lipschitz coefficients (and for an obstacle problem without sign assumption on the solution) by Caffarelli, Shahgholian [16]. For the classical obstacle problem, pointwise regularity results of the singular set have been obtained for double Dini coefficients in Monneau [32]. This result was based on a monotonicity formula devoted to singular points (see also Monneau [33]). In the proof of Theorem 1.10, this monotonicity formula for singular points has been refined, which allows us to get the result assuming the modulus of continuity to be only Dini.

The proof of theorems 1.2, 1.8 and 1.11 are based on a decay estimate (see Propositions 2.5, 6.2 and 7.4), similar to other decay estimates obtained for dynamical systems converging to stable states (see for instance Simon [35]). Our proof of this decay estimate is done by contradiction, and uses blow-up techniques like in Caffarelli [7] and the stability of the obstacle problem. Our approach is strongly inspired on the one hand from the epiperimetric inequality given in Weiss [38], and on the other hand on blow-up techniques and Caccioppoli inequalities as used in Evans [22], Evans, Gariepy [23], and finally on classical results for Dini-continuity results for solutions of elliptic equations or systems (see the references cited in subsection 1.1).

Remark 1.14 From our proofs, we can check that all the previous estimates are still true (with different constants), if we replace $\sigma_{p}(s)$ by $\sigma_{p}(\gamma s)$ for a fixed constant $\gamma \in(0,1]$.

\subsection{Organization of the article}

In Section 2, we prove a fundamental decay estimate (Proposition 2.5) for the Laplace equation and give the proof of a weak version of Theorem 1.2, namely Theorem 2.1, and finally give the proof of Theorem 1.2. The proof of the Theorem also uses some general large scales estimates (Lemma 2.9 and Proposition 2.7) that are proved in Section 3.

In Section 4, we start the study of the obstacle problem, giving the proof of the growth estimate Proposition 1.5 and the monotonicity formula Theorem 1.6. In Section 5, we study degenerate points of the free boundary and give an example of such points. In Section 6 , we study the regular points of the free boundary and prove Theorem 1.8, also based on a decay estimate (Proposition 6.2).

Section 7 is devoted to the study of singular points of the free boundary. We first prove two monotonicity formulas (one for double Dini coefficients and another one for Dini coefficients assuming moreover $p \geq 2$ ). We then show Theorem 1.10 on the uniqueness of the blow-up limits. The quantitative estimate Theorem 1.11 is proved using a decay estimate. This decay 
estimate (Proposition 7.4) is in particular based on new Liouville type results.

In Section 8, we give some applications to the regularity of the free boundary for general second order linear elliptic operators. In the Appendix (Section 9), we also give an application to the regularity of solutions to fully nonlinear elliptic equations.

\section{A decay estimate for Laplace equation and proof of Theorem 1.2}

\subsection{Proof of a weak version of Theorem 1.2}

We will start to prove a weak version of Theorem 1.2 (namely Theorem 2.1) whose proof is slightly simpler and enlights the method we use. Moreover this method of proof will be directly adapted later for the obtacle problem. The proof of Theorem 1.2 will be done in Subsection 2.4 and will consists in an adaptation of the proof of the following result:

Theorem 2.1 (Pointwise modulus of continuity for the Laplace equation)

Let $p \in(1,+\infty)$ given. Then there exist $\alpha \in(0,1]$ and constants $C>0, r_{0} \in(0,1)$, such that, given a function $u \in L^{p}\left(B_{1}\right)$ satisfying (1.1) with a modulus of continuity $\sigma_{p}$ defined in (1.9) which is assumed Dini, then there exists a harmonic polynomial $P_{0}$ of degree less or equal to 2 , such that for every $r \in\left(0, r_{0}\right)$ :

$$
\left(\frac{1}{\left|B_{r}\right|} \int_{B_{r}}\left|\frac{u(x)-P_{0}(x)}{r^{2}}\right|^{p}\right)^{\frac{1}{p}} \leq C\left(M_{0} r^{\alpha}+\int_{0}^{r} \frac{\sigma_{p}(s)}{s} d s+r^{\alpha} \int_{r}^{1} \frac{\sigma_{p}(s)}{s^{1+\alpha}} d s\right)
$$

and

$$
P_{0}(x)=a+b \cdot x+\frac{1}{2} t x \cdot c \cdot x
$$

with

$$
|a|+|b|+|c| \leq C M_{0} \quad \text { and } \quad M_{0}=\int_{0}^{1} \frac{\sigma_{p}(s)}{s} d s+\left(\int_{B_{1}}|u|^{p}\right)^{\frac{1}{p}}
$$

For the reader's convenience, we recall the equation (1.1) satisfied by $u$, namely

$$
\left\{\begin{array}{l}
\Delta u=f \quad \text { on } \quad B_{1} \\
f \in L^{p}\left(B_{1}\right) \text { and } \quad f(0)=0
\end{array}\right.
$$

We will use the following

\section{Definition 2.2 (Quantities $M$ and $N$ )}

We introduce the following set of functions

$\mathcal{P}_{2}=\{P, \quad$ with $\quad P \quad$ polynomial of degree less or equal to 2 such that $\Delta P=0\}$ and define

$$
M(u, \rho)=\sup _{r \in(0, \rho]} N(u, r) \quad \text { with } \quad N(u, r)=\inf _{P \in \mathcal{P}_{2}}\left(\frac{1}{r^{n+2 p}} \int_{B_{r}}|u-P|^{p}\right)^{\frac{1}{p}}
$$

When there is no ambiguity on the choice of function $u$, we simply denote these quantities by $M(\rho)$ and $N(r)$. 
Let us remark that $M$ and $N$ give a measure of the distance between the function $u$ and the set $\mathcal{P}_{2}$ which contains the possible limit behaviour of the solution at the origin.

At this stage, it is not clear if $M$ is finite or not. Nevertheless, we have the following property which will be proved at the end of Subsection 2.3. We claim the following

\section{Proposition 2.3 (Finiteness of $M$ )}

There exists a constant $C>0$ such that

$$
\forall r \in(0,1), \quad M(u, r) \leq C\left\{\left(\int_{B_{1}}|u|^{p}\right)^{\frac{1}{p}}+\sigma_{p}(1)\right\}
$$

Remark 2.4 Proposition 2.3 is sharp, in view of the following example. In dimension $n=2$, let us consider $P(x)=x_{1}^{2}-x_{2}^{2}$ for $x=\left(x_{1}, x_{2}\right)$. Then $u(x)=P(x) \ln |x|$ satisfies $\Delta u(x)=4 P(x) /|x|^{2}$. Therefore $\Delta u$ is bounded, while $D^{2} u$ is not.

Then we have the following cornerstone result which will be proved in Subsection 2.3.

\section{Proposition 2.5 (Decay estimate in a smaller ball)}

Given $p \in(1,+\infty)$, there exist constants $C_{0}>0, r_{0}, \lambda, \mu \in(0,1)$ (only depending on $p$ and the dimension $n$ ) such that for every functions $u$ and $f$ satisfying (1.1) with a modulus of continuity $\sigma_{p}$ given by (1.9), then we have the following property

$$
\forall r \in\left(0, r_{0}\right), \quad M(u, \lambda r)<\mu M(u, r) \quad \text { or } \quad M(u, r)<C_{0} \sigma_{p}(r / 2)
$$

Remark 2.6 Here the problem is linear, so $M(u, r)$ does not need to be small to satisfy the decay estimate.

Contrarily to Proposition 2.5, the following result does not depend on the particular PDE that we study, but can be considered as a routine result and will be proved in Section 3 .

\section{Proposition 2.7 (Modulus of continuity of the solution up to the second order)}

Let us consider any function $u$ which satisfies (2.17) with constants $C_{0}>0, r_{0}, \lambda, \mu \in(0,1)$, and a Dini modulus of continuity $\sigma_{p}$. Let us define $\alpha=\ln \mu / \ln \lambda$. Then there exist $P_{0} \in \mathcal{P}_{2}$ and a constant $C_{0}^{\prime}>0$ only depending on $C_{0}, r_{0}, \lambda, \mu$, such that for every $\rho \in\left(0, \lambda r_{0} / 2\right)$, we have

$$
\left(\frac{1}{\rho^{n+2 p}} \int_{B_{\rho}} d y\left|u-P_{0}\right|^{p}\right)^{\frac{1}{p}} \leq C_{0}^{\prime}\left\{M\left(u, r_{0}\right) \rho^{\alpha}+\int_{0}^{\rho} \frac{\sigma_{p}(r)}{r} d r+\rho^{\alpha} \int_{\rho}^{r_{0}} \frac{\sigma_{p}(r)}{r^{1+\alpha}} d r\right\}
$$

\section{Proof of Theorem 2.1}

The proof of Theorem 2.1 follows from estimates on $M\left(u, r_{0}\right)$ and on $P_{0}$ that we establish successively.

Estimate on $M\left(u, r_{0}\right)$

Because the right hand side of the inequality (2.18) is non-increasing with $\alpha$, it is sufficient to replace $\alpha$ by $\min (1, \alpha)$. Let us define $r_{1}$ such that $C_{0}^{\prime} r_{1}^{\alpha}=1 / 2$. Then we have

$$
M\left(u, r_{0}\right) \leq M\left(u, r_{1}\right)+\sup _{\rho \in\left[r_{1}, r_{0}\right]}\left(\frac{1}{\rho^{n+2 p}} \int_{B_{\rho}}|u|^{p}\right)^{\frac{1}{p}}
$$


and

$$
M\left(u, r_{1}\right)=N\left(u, \rho_{0}\right) \leq\left(\frac{1}{\rho_{0}^{n+2 p}} \int_{B_{\rho_{0}}}\left|u-P_{0}\right|^{p}\right)^{\frac{1}{p}}
$$

for some $\rho_{0} \in\left(0, r_{1}\right]$ for which we deduce from $(2.18)$ that

$$
M\left(u, r_{1}\right) \leq 2 C_{0}^{\prime}\left\{\int_{0}^{\rho_{0}} \frac{\sigma_{p}(r)}{r} d r+\rho_{0}^{\alpha} \int_{\rho_{0}}^{r_{0}} \frac{\sigma_{p}(r)}{r^{1+\alpha}} d r\right\}
$$

Because the right hand side is a non-decreasing function of $\rho_{0}$, we deduce that there exists a constant $C_{1}>0$ only depending on $C_{0}^{\prime}, r_{0}, \alpha, n, p$ such that

$$
M\left(u, r_{0}\right) \leq C_{1}\left(\int_{0}^{r_{0}} \frac{\sigma_{p}(r)}{r} d r+\left(\int_{B_{r_{0}}}|u|^{p}\right)^{\frac{1}{p}}\right)
$$

\section{Estimate on $P_{0}$}

Let us remark that for some $\rho_{0}$ (for instance $\rho_{0}=\lambda r_{0} / 4$ ) we have

$$
\left(\frac{1}{\rho_{0}^{n+2 p}} \int_{B_{\rho_{0}}}\left|P_{0}\right|^{p}\right)^{\frac{1}{p}} \leq\left(\frac{1}{\rho_{0}^{n+2 p}} \int_{B_{\rho_{0}}}|u|^{p}\right)^{\frac{1}{p}}+\left(\frac{1}{\rho_{0}^{n+2 p}} \int_{B_{\rho_{0}}}\left|u-P_{0}\right|^{p}\right)^{\frac{1}{p}}
$$

Then from (2.18), we deduce that for some constant $C_{2}>0$

$$
\left(\frac{1}{\rho_{0}^{n+2 p}} \int_{B_{\rho_{0}}}\left|P_{0}\right|^{p}\right)^{\frac{1}{p}} \leq C_{2}\left(\int_{0}^{\rho_{0}} \frac{\sigma_{p}(r)}{r} d r+\left(\int_{B_{\rho_{0}}}|u|^{p}\right)^{\frac{1}{p}}\right)
$$

Finally, if $P_{0}(x)=a+b \cdot x+\frac{1}{2} t \cdot c \cdot x$, it can be easily checked that there exists a constant $C_{3}$ (independent on $P_{0}$ ) such that

$$
|a|+|b|+|c| \leq C_{3}\left(\frac{1}{\rho_{0}^{n+2 p}} \int_{B_{\rho_{0}}}\left|P_{0}\right|^{p}\right)^{\frac{1}{p}}
$$

This implies the result and ends the proof of the Theorem.

\subsection{Preliminary results}

Before to perform the proof of Proposition 2.5, we need two lemmata. We first state and prove the following Caccioppoli type estimate.

\section{Lemma 2.8 (Caccioppoli type estimate)}

Let $\zeta \in C_{0}^{\infty}\left(\mathbb{R}^{n}\right)$ with supp $\zeta \subset B_{R}(0)$ with $R>0$. Let $P \in \mathcal{P}_{2}$ and $u$ be a solution of (1.1) and $\sigma_{p}$ defined in (1.9) for some $p \in(1,+\infty)$. Then we have for $W=(u-P)|u-P|^{\frac{p}{2}-1}$ and $\frac{1}{p}+\frac{1}{p^{\prime}}=1$

$$
\int_{\mathbb{R}^{n}} \frac{4(p-1)}{p^{2}} \zeta^{2}|\nabla W|^{2} \leq \int_{\mathbb{R}^{n}} \frac{4}{p-1} W^{2}|\nabla \zeta|^{2}+2\left|B_{R}\right| \sigma_{p}(R)\left(\frac{1}{\left|B_{R}\right|} \int_{B_{R}} \zeta^{2 p^{\prime}}|W|^{2}\right)^{\frac{1}{p^{\prime}}}
$$




\section{Proof of Lemma 2.8}

On the ball $B_{1}$, we have

$$
-\Delta u+f=0 \quad \text { and } \quad-\Delta P=0=f(0)
$$

for every $P \in \mathcal{P}$. Taking the difference of the equations, and multiplying by $\zeta^{2} w|w|^{p-2}$ for $w=u-P$, we get

$$
\int_{\mathbb{R}^{n}}-\zeta^{2} w|w|^{p-2} \Delta w=\int_{B_{R}}-\zeta^{2} w|w|^{p-2}(f(x)-f(0))
$$

An integration by parts shows that we get with $W=w|w|^{\frac{p}{2}-1}$

$$
\int_{\mathbb{R}^{n}}-\zeta^{2} w|w|^{p-2} \Delta w=\int_{\mathbb{R}^{n}} \frac{4(p-1)}{p^{2}} \zeta^{2}|\nabla W|^{2}+\frac{4}{p} \zeta W \nabla \zeta \cdot \nabla W
$$

Therefore for $\lambda=\frac{4(p-1)}{p^{2}}$, we get with $\frac{1}{p}+\frac{1}{p^{\prime}}=1$

$$
\begin{aligned}
& \int_{\mathbb{R}^{n}} \lambda \zeta^{2}|\nabla W|^{2} \\
& \leq \int_{\mathbb{R}^{n}}-\frac{4}{p} \zeta W \nabla \zeta \cdot \nabla W+\int_{B_{R}} \zeta^{2}|W|^{\frac{2(p-1)}{p}}|f(x)-f(0)| \\
& \leq \int_{\mathbb{R}^{n}} \frac{1}{2}\left\{\lambda \zeta^{2}|\nabla W|^{2}+\frac{16}{p^{2}} \lambda^{-1} W^{2}|\nabla \zeta|^{2}\right\}+\left|B_{R}\right| \sigma_{p}(R)\left(\frac{1}{\left|B_{R}\right|} \int_{B_{R}}\left(\zeta^{2}|W|^{\frac{2(p-1)}{p}}\right)^{p^{\prime}}\right)^{\frac{1}{p^{\prime}}} \\
& \leq \int_{\mathbb{R}^{n}} \frac{1}{2}\left\{\lambda \zeta^{2}|\nabla W|^{2}+\frac{4}{p-1} W^{2}|\nabla \zeta|^{2}\right\}+\left|B_{R}\right| \sigma_{p}(R)\left(\frac{1}{\left|B_{R}\right|} \int_{B_{R}} \zeta^{2 p^{\prime}}|W|^{2}\right)^{\frac{1}{p^{\prime}}}
\end{aligned}
$$

Substracting the term $\frac{1}{2} \int_{\mathbb{R}^{n}} \lambda \zeta^{2}|\nabla W|^{2}$ to the left hand side, this gives (2.19). This ends the proof of the Lemma.

We will also use the following result which shows that we can control a distance between the function $u$ and a particular element of $\mathcal{P}_{2}$, once we control an integral of $M(u, s) / s$ :

Lemma 2.9 (Control of $u$ by $M$ )

Let us assume that the set $\mathcal{P}$ is either the set of harmonic polynomials of degree less or equal to 2 , or that each element of $\mathcal{P}$ is homogeneous of degree 2.

For $p \in(1,+\infty)$, there exists a constant $C_{1}>0$ (which only depends on $p$ and the dimension n) such that if

$$
N(u, 1)=\left(\int_{B_{1}}\left|u-P_{1}\right|^{p}\right)^{\frac{1}{p}} \text { for some } P_{1} \in \mathcal{P}
$$

and if $u$ is defined on $B_{2 \rho}$ with $\rho \geq 1$, then we have

$$
\left(\frac{1}{\rho^{n+2 p}} \int_{B_{\rho}}\left|u-P_{1}\right|^{p}\right)^{\frac{1}{p}} \leq C_{1} \int_{1}^{2 \rho} \frac{M(u, s)}{s} d s
$$

This result will be proved in Section 3 . 


\subsection{Proof of the decay estimate Proposition 2.5}

\section{Proof of Proposition 2.5}

We perform the proof by contradiction in two Steps. For simplicity, we fix the exponent $p$, and set

$$
\sigma(r)=\sigma_{p}(r / 2)
$$

By the way, we will have to consider sequences of modulus of continuity $\sigma$ that we denote by $\left(\sigma_{m}\right)_{m}$ indexed by $m$, with no possible confusion.

\section{Step 1: A priori estimates on a sequence $v_{m}$}

If the Proposition is false, then there exist sequences $\left(r_{m}\right)_{m},\left(C_{m}\right)_{m},\left(\lambda_{m}\right)_{m},\left(\mu_{m}\right)_{m},\left(f_{m}\right)_{m}$, $\left(u_{m}\right)_{m},\left(\sigma_{m}\right)_{m}$ such that

$$
\left\{\begin{array}{l}
r_{m}, \lambda_{m} \longrightarrow 0 \\
C_{m} \longrightarrow+\infty \\
\mu_{m} \longrightarrow 1
\end{array}\right.
$$

and

$$
M\left(u_{m}, r_{m}\right) \geq C_{m} \sigma_{m}\left(r_{m}\right) \text { and } M\left(u_{m}, \lambda_{m} r_{m}\right) \geq \mu_{m} M\left(u_{m}, r_{m}\right)
$$

From Proposition 2.3, $M\left(u_{m}, \cdot\right)$ is bounded (and non-decreasing). Therefore there exists $\rho_{m} \in\left(0, \lambda_{m} r_{m}\right]$, such that $N\left(u_{m}, \rho_{m}\right)$ is arbitrarily close to $M\left(u_{m}, \lambda_{m} r_{m}\right)$ and satisfies for instance

$$
\frac{M\left(u_{m}, \lambda_{m} r_{m}\right)}{1+1 / m} \leq N\left(u_{m}, \rho_{m}\right)=: \varepsilon_{m}
$$

with

$$
N\left(u_{m}, \rho_{m}\right)=\left(\frac{1}{\rho_{m}^{n+2 p}} \int_{B_{\rho_{m}}}\left|u_{m}-P_{m}\right|^{p}\right)^{\frac{1}{p}} \quad \text { for some } \quad P_{m} \in \mathcal{P}_{2}
$$

We now apply Lemma 2.9 to $u_{m}^{\rho_{m}}(x)=u_{m}\left(\rho_{m} \cdot x\right) / \rho_{m}^{2}, P_{m}^{\rho_{m}}(x)=P_{m}\left(\rho_{m} \cdot x\right) / \rho_{m}^{2}$ and get for every $s \in\left(1, s_{m} / 2\right)$ with $s_{m}=r_{m} / \rho_{m} \geq 1 / \lambda_{m} \longrightarrow+\infty$ :

$$
\begin{aligned}
\left(\frac{1}{\left(s \rho_{m}\right)^{n+2 p}} \int_{B_{s \rho_{m}}}\left|u_{m}-P_{m}\right|^{p}\right)^{\frac{1}{p}} & =\left(\frac{1}{s^{n+2 p}} \int_{B_{s}}\left|u_{m}^{\rho_{m}}-P_{m}^{\rho_{m}}\right|^{p}\right)^{\frac{1}{p}} \\
& \leq C_{1} \int_{1}^{2 s} d s^{\prime} \frac{M\left(u_{m}^{\rho_{m}}, s^{\prime}\right)}{s^{\prime}} \\
& \leq C_{1} \int_{1}^{2 s} d s^{\prime} \frac{M\left(u_{m}, s^{\prime} \rho_{m}\right)}{s^{\prime}} \\
& \leq C_{1} \frac{(1+1 / m) \varepsilon_{m}}{\mu_{m}} \ln (2 s)
\end{aligned}
$$

We now define the renormalized function

$$
v_{m}(y):=\frac{1}{\varepsilon_{m} \rho_{m}^{2}}\left(u_{m}-P_{m}\right)\left(\rho_{m} y\right)
$$


which satisfies

$$
\Delta v_{m}=g_{m} \quad \text { with } \quad g_{m}(y)=\frac{f_{m}\left(\rho_{m} y\right)}{\varepsilon_{m}}
$$

with for fixed $R>0$

$$
\left(\frac{1}{\left|B_{R}\right|} \int_{B_{R}}\left|g_{m}\right|^{p}\right)^{\frac{1}{p}}=\frac{\sigma_{m}\left(2 \rho_{m} R\right)}{\varepsilon_{m}} \longrightarrow 0 \quad \text { as } \quad m \longrightarrow+\infty
$$

Indeed, from (2.20), for $s \in\left(0, s_{m}\right)$, we deduce that

$$
\sigma_{m}\left(s \rho_{m}\right) \leq \sigma_{m}\left(s_{m} \rho_{m}\right)=\sigma_{m}\left(r_{m}\right) \leq \frac{\varepsilon_{m}(1+1 / m)}{\mu_{m} C_{m}}
$$

and therefore for every $s \in\left(0, s_{m}\right)$ we have

$$
\frac{\sigma_{m}\left(\rho_{m} s\right)}{\varepsilon_{m}} \leq \frac{1+1 / m}{\mu_{m} C_{m}} \longrightarrow 0
$$

Moreover we have (because here $\mathcal{P}_{2}$ is a vector space)

$$
\inf _{P \in \mathcal{P}_{2}}\left(\int_{B_{1}}\left|v_{m}-P\right|^{p}\right)^{\frac{1}{p}}=1
$$

and for $s \in\left(1, s_{m} / 2\right)$

$$
\left(\frac{1}{s^{n+2 p}} \int_{B_{s}}\left|v_{m}\right|^{p}\right)^{\frac{1}{p}} \leq \frac{C_{1}(1+1 / m)}{\mu_{m}} \ln (2 s) \longrightarrow C_{1} \ln (2 s)
$$

where the limits are taken as $m$ goes to infinity.

We now apply the Caccioppoli type estimate (2.19) to $v_{m}$, we get for $\operatorname{supp} \zeta \subset B_{R}$ and $W_{m}=v_{m}\left|v_{m}\right|^{\frac{p}{2}-1}$

$$
\int_{\mathbb{R}^{n}} \frac{4(p-1)}{p^{2}} \zeta^{2}\left|\nabla W_{m}\right|^{2} \leq \int_{\mathbb{R}^{n}} \frac{4}{p-1} W_{m}^{2}|\nabla \zeta|^{2}+2\left|B_{R}\right| \frac{\sigma_{m}\left(2 \rho_{m} R\right)}{\varepsilon_{m}}\left(\frac{1}{\left|B_{R}\right|} \int_{B_{R}} \zeta^{2 p^{\prime}}\left|W_{m}\right|^{2}\right)^{\frac{1}{p^{\prime}}}
$$

\section{Step 2: Convergence of the sequence $v_{m}$}

From $(2.22),(2.26)$ and $(2.25)$, we get that for every $R>0$, there exists a constant $C_{R}>0$ such that uniformly in $m$ we have

$$
\left|W_{m}\right|_{H^{1}\left(B_{R}\right)} \leq C_{R}
$$

Then up to extract a subsequence, we can assume that

$$
\begin{cases}W_{m} \longrightarrow W_{\infty}=v_{\infty}\left|v_{\infty}\right|^{\frac{p}{2}-1} & \text { in } L_{l o c}^{2}\left(\mathbb{R}^{n}\right) \text { and a.e. in } \mathbb{R}^{n} \\ W_{m} \longrightarrow W_{\infty} & \text { weakly in } H_{l o c}^{1}\left(\mathbb{R}^{n}\right)\end{cases}
$$

where $v_{\infty}$ has to be seen as the limit of $v_{m}$, and (using for instance the dominated convergence Theorem of Lebesgue)

$$
\inf _{P \in \mathcal{P}_{2}}\left(\int_{B_{1}}\left|v_{\infty}-P\right|^{p}\right)^{\frac{1}{p}}=1
$$


and

$$
\left(\frac{1}{s^{n+2 p}} \int_{B_{s}}\left|v_{\infty}\right|^{p}\right)^{\frac{1}{p}} \leq C_{1} \ln (2 s) \quad \text { for every } \quad s \geq 1
$$

and by (2.22), we deduce that $v_{\infty}$ satisfies

$$
\Delta v_{\infty}=0 \quad \text { on } \quad \mathbb{R}^{n}
$$

Together with (2.28), we see that $v_{\infty} \in \mathcal{S}^{\prime}$ (the dual of the Schwarz space) and then $v_{\infty}$ is a polynomial, whose degree is less or equal to 2 by (2.28). Therefore this is in contradiction with (2.27).

This ends the proof of the Proposition.

\section{Proof of Proposition 2.3}

We will do the proof in two steps. For simplicity, we fix the exponent $p$, and set

$$
\sigma(r)=\sigma_{p}(r)
$$

By the way, we will have to consider sequences of modulus of continuity $\sigma$ that we denote by $\left(\sigma_{m}\right)_{m}$ indexed by $m$, with no possible confusion.

Step 1: finiteness of $M(u, \cdot)$

The proof of the finiteness of $M(u, \cdot)$ follows almost lines by lines the proof of Proposition 2.5 for the decay estimate on $M$.

Assumme that $M\left(u, r_{0}\right)=+\infty$ for some $r_{0} \in(0,1)$. This implies that there is a sequence $\left(\rho_{m}\right)_{m}$ such that

$$
N\left(u, \rho_{m}\right) \longrightarrow+\infty \quad \text { with } \quad \rho_{m} \longrightarrow 0
$$

and

$$
N(u, r) \leq N\left(u, \rho_{m}\right) \quad \text { for } \quad r \in\left(\rho_{m}, r_{0}\right)
$$

Still defining $\varepsilon_{m}=N\left(u, \rho_{m}\right)$ (which this times goes to infinity), we see that

$$
v_{m}(y):=\frac{1}{\varepsilon_{m} \rho_{m}^{2}}\left(u-P_{m}\right)\left(\rho_{m} y\right)
$$

still satisfies

$$
\inf _{P \in \mathcal{P}_{2}}\left(\int_{B_{1}}\left|v_{m}-P\right|^{p}\right)^{\frac{1}{p}}=1
$$

and for $s \in\left(1, s_{m} / 2\right)$ with this time $s_{m}=r_{0} / \rho_{m} \rightarrow+\infty$

$$
\left(\frac{1}{s^{n+2 p}} \int_{B_{s}}\left|v_{m}\right|^{p}\right)^{\frac{1}{p}} \leq C_{1} \ln (2 s)
$$

where we have used the fact that the maximum of $M(u, \cdot)$ is reached at $\rho_{m}$ in $(2.21)$, and the Caccioppoli type estimate for $\operatorname{supp} \zeta \subset B_{R}$ and $W_{m}=v_{m}\left|v_{m}\right|^{\frac{p}{2}-1}$

$$
\int_{\mathbb{R}^{n}} \frac{4(p-1)}{p^{2}} \zeta^{2}\left|\nabla W_{m}\right|^{2} \leq \int_{\mathbb{R}^{n}} \frac{4}{p-1} W_{m}^{2}|\nabla \zeta|^{2}+2\left|B_{R}\right| \frac{\sigma\left(R \rho_{m}\right)}{\varepsilon_{m}}\left(\frac{1}{\left|B_{R}\right|} \int_{B_{R}} \zeta^{2 p^{\prime}}\left|W_{m}\right|^{2}\right)^{\frac{1}{p^{\prime}}}
$$


where we see directly this time that $\frac{\sigma\left(R \rho_{m}\right)}{\varepsilon_{m}} \rightarrow 0$, because $\varepsilon_{m} \rightarrow+\infty$ and $\sigma$ is assumed finite. Finally, using the fact that $\varepsilon_{m} \rightarrow+\infty$, we get that the limit $v_{\infty}$ of $v_{m}$ is harmonic, and we get the contradiction following Step 2 of the proof of Proposition 2.5.

Step 2: bound on $M(u, \cdot)$

We now know that $M(u, \cdot)$ is bounded. Let us assume that the Proposition is false. Again, we can find sequences $\left(r_{m}\right)_{m},\left(C_{m}\right)_{m},\left(f_{m}\right)_{m},\left(u_{m}\right)_{m},\left(\sigma_{m}\right)_{m}$ such that

$$
C_{m} \longrightarrow+\infty
$$

and

$$
M\left(u_{m}, r_{m}\right) \geq C_{m}\left\{\left(\int_{B_{1}}\left|u_{m}\right|^{p}\right)^{\frac{1}{p}}+\sigma_{m}(1)\right\}
$$

Therefore there exists $\rho_{m} \in\left(0, r_{m}\right]$, such that $N\left(u_{m}, \rho_{m}\right)$ is arbitrarily close to $M\left(u_{m}, r_{m}\right)$. Therefore, we have $\rho_{m} \longrightarrow 0$ (because $N\left(u_{m}, r\right)$ is bounded by $C_{r_{0}}\left(\int_{B_{1}}\left|u_{m}\right|^{p}\right)^{\frac{1}{p}}$ for some constant $C_{r_{0}}>0$ for $r \geq r_{0}>0$ ). Consequently, we can choose $\rho_{m}$ satisfying for instance

$$
\frac{M\left(u_{m}, r_{m}\right)}{1+1 / m} \leq N\left(u_{m}, \rho_{m}\right)=: \varepsilon_{m}
$$

and

$$
N(u, r) \leq N\left(u, \rho_{m}\right) \quad \text { for } \quad r \in\left(\rho_{m}, 1\right)
$$

We finally proceed as in Step 1 of the present proof, but defining

$$
v_{m}(y):=\frac{1}{\varepsilon_{m} \rho_{m}^{2}}\left(u_{m}-P_{m}\right)\left(\rho_{m} y\right)
$$

Moreover in $(2.30), C_{1}$ is replaced by $C_{1}(1+1 / m)$, and in $(2.31), \frac{\sigma\left(R \rho_{m}\right)}{\varepsilon_{m}}$ can be replaced by $\frac{\sigma_{m}(1)}{\varepsilon_{m}} \leq \frac{1+1 / m}{C_{m}} \rightarrow 0$. This ends the proof of Proposition 2.3.

\subsection{Proof of Theorem 1.2}

The proof of Theorem 1.2 is an adaptation of the proofs of Proposition 2.3 and Theorem 2.1. We give below the results that we have to adapt.

First, we define a (non-unique) constant $c_{r}$ such that

$$
\inf _{c \in \mathbb{R}} \int_{B_{r}}|f(x)-c|^{p}=\frac{1}{\left|B_{r}\right|} \int_{B_{r}}\left|f(x)-c_{r}\right|^{p} .
$$

Then, we fix a polynomial $P_{*}$ homogeneous of degree 2 satisfying $\Delta P_{*}=1$ (for instance $\left.P_{*}(x)=\frac{x^{2}}{2 n}\right)$, and define

$$
\hat{M}(u, \rho)=\sup _{r \in(0, \rho]} \hat{N}(u, r) \quad \text { with } \quad \hat{N}(u, r)=\inf _{P \in \mathcal{P}_{2}}\left(\frac{1}{r^{n+2 p}} \int_{B_{r}}\left|u-P-c_{r} P_{*}\right|^{p}\right)^{\frac{1}{p}}
$$


where $\mathcal{P}_{2}$ is still the set of harmonic polynomials of degree less or equal to 2 . The renormalized function is then

$$
v_{m}(y):=\frac{1}{\varepsilon_{m} \rho_{m}^{2}}\left(u-P_{m}-c_{\rho_{m}} P_{*}\right)\left(\rho_{m} y\right)
$$

with $P_{m} \in \mathcal{P}_{2}$ realizing the infimum in the definition of $\hat{N}\left(u, \rho_{m}\right)$.

Again, assuming first that $\hat{M}(u, \cdot)$ is finite, we prove a decay estimate similar to Proposition 2.5, with $M(u, \cdot)$ and $\sigma_{p}$ respectively replaced by $\hat{M}(u, \cdot)$ and $\tilde{\sigma}_{p}$. To this end, we check easily that the proof of Lemma 2.9 applies perfectly in our case. Indeed, it is sufficient to work with the polynomials $\hat{P}_{r}=P_{r}+c_{r} P_{*}$ which gives with the same constant $C_{1}$ the result for $\rho \geq 1$ (and then for $u$ rescalled):

$$
\left(\frac{1}{\rho^{n+2 p}} \int_{B_{\rho}}\left|u-P_{1}-c_{1} P_{*}\right|^{p}\right)^{\frac{1}{p}} \leq C_{1} \int_{1}^{2 \rho} \frac{\hat{M}(u, s)}{s} d s
$$

Then the rest of the proof is similar with the choice

$$
g_{m}(y)=\frac{f_{m}\left(\rho_{m} y\right)-c_{\rho_{m}}}{\varepsilon_{m}}
$$

The proof of the analogue of Proposition 2.3 is the same, except that here $N\left(u_{m}, r\right)$ is bounded by $C_{r_{0}}\left(\left(\int_{B_{1}}\left|u_{m}\right|^{p}\right)^{\frac{1}{p}}+\left(\int_{B_{1}}\left|f_{m}\right|^{p}\right)^{\frac{1}{p}}\right)$ for some constant $C_{r_{0}}>0$ for $1 \geq r \geq r_{0}>$ 0 .

Lemmata 3.3 and 3.4 are still true with $M(u, \cdot)$ and $\sigma_{p}$ replaced by $\hat{M}(u, \cdot)$ and $\tilde{\sigma}_{p}$. Noticing that

$$
\tilde{M}(u, r) \leq \hat{M}(u, r),
$$

we get (1.7) (i.e. the analogue of Proposition 2.7) as previously as a consequence of Lemma 3.5 applied to $\tilde{M}(u, \cdot)$ with $\mathcal{P}=\tilde{\mathcal{P}}_{2}$.

Finally, in the rest of the proof of Theorem 1.2 consisting to control the coefficients of $P_{0}$, the only change appears applying (1.7) instead of (2.18). Therefore we use the bound (1.6) to get the estimate on the coefficients of the polynomial. This makes appear an additional term $\left(\int_{B_{1}}|f|^{p}\right)^{\frac{1}{p}}$, and finishes the proof of Theorem 1.2.

\section{General large scale estimates : proof of Lemma 2.9 and Proposition 2.7}

\subsection{Proof of Lemma 2.9}

Before to prove Lemma 2.9, we need the following easy result:

\section{Lemma 3.1 (Larger ball/smaller ball)}

There exists a constant $C_{2} \geq 1$ only depening on $p$ and the dimension $n$ such that for every 
polynomial $P$ of degree less or equal to 2 , we have for any $r \geq 1$ :

$$
\left(\frac{1}{r^{n+2 p}} \int_{B_{r}}|P|^{p}\right)^{\frac{1}{p}} \leq C_{2}\left(\int_{B_{1}}|P|^{p}\right)^{\frac{1}{p}}
$$

\section{Proof of Lemma 3.1}

We simply remark that if $P(x)=a+b \cdot x+\frac{1}{2} t x \cdot c \cdot x$, then there exists a constant $C_{0}>0$ such that

$$
\left(\frac{1}{r^{n+2 p}} \int_{B_{r}}|P|^{p}\right)^{\frac{1}{p}} \leq C_{0}\left(\frac{|a|}{r^{2}}+\frac{|b|}{r}+|c|\right)
$$

On the other hand there exists a constant $C_{1}>0$ (easily checked by contradiction) such that

$$
|a|+|b|+|c| \leq C_{1}\left(\int_{B_{1}}|P|^{p}\right)^{\frac{1}{p}}
$$

Putting together these two inequalities, we get the result for $r \geq 1$, which ends the proof of the Lemma.

\section{Proof of Lemma 2.9}

For every $r>0$, we have

$$
N(r)=\left(\frac{1}{r^{n+2 p}} \int_{B_{r}}\left|u-P_{r}\right|^{p}\right)^{\frac{1}{p}} \text { for some } \quad P_{r} \in \mathcal{P}
$$

Then for $\alpha \in(1,2]$ we have

$$
\begin{aligned}
\left(\frac{1}{r^{n+2 p}} \int_{B_{r}}\left|P_{\alpha r}-P_{r}\right|^{p}\right)^{\frac{1}{p}} & \leq\left(\frac{1}{r^{n+2 p}} \int_{B_{r}}\left|u-P_{r}\right|^{p}\right)^{\frac{1}{p}}+\left(\frac{1}{r^{n+2 p}} \int_{B_{r}}\left|u-P_{\alpha r}\right|^{p}\right)^{\frac{1}{p}} \\
& \leq\left(\frac{1}{r^{n+2 p}} \int_{B_{r}}\left|u-P_{r}\right|^{p}\right)^{\frac{1}{p}}+\alpha^{\frac{n+2 p}{p}}\left(\frac{1}{(\alpha r)^{n+2 p}} \int_{B_{\alpha r}(0)}\left|u-P_{\alpha r}\right|^{p}\right)^{\frac{1}{p}} \\
& \leq \alpha^{\frac{n+2 p}{p}}(N(r)+N(\alpha r)) \\
& \leq C_{0} M(\alpha r) \text { with } C_{0}=2^{\frac{n+3 p}{p}}
\end{aligned}
$$

In the case where the elements $P \in \mathcal{P}$ are 2-homogeneous, i.e. satisfy $P(r x)=r^{2} P(x)$, we simply have

$$
\left(\int_{B_{1}}\left|P_{2 r}-P_{r}\right|^{p}\right)^{\frac{1}{p}}=\left(\frac{1}{r^{n+2 p}} \int_{B_{r}}\left|P_{2 r}-P_{r}\right|^{p}\right)^{\frac{1}{p}} \leq C_{0} M(2 r)
$$

In the case where $\mathcal{P}$ is the set of harmonic polynomials of degree less or equal to 2 , we deduce from (3.32) and a rescaling that for $\rho \geq r>0$ :

$$
\left(\frac{1}{\rho^{n+2 p}} \int_{B_{\rho}}\left|P_{2 r}-P_{r}\right|^{p}\right)^{\frac{1}{p}} \leq C_{2}\left(\frac{1}{r^{n+2 p}} \int_{B_{r}}\left|P_{2 r}-P_{r}\right|^{p}\right)^{\frac{1}{p}} \leq C M(2 r) \quad \text { with } \quad C=C_{2} C_{0}
$$


Similarly, we get

$$
\left(\frac{1}{\rho^{n+2 p}} \int_{B_{\rho}}\left|P_{r}-P_{1}\right|^{p}\right)^{\frac{1}{p}} \leq C_{2}\left(\frac{1}{r^{n+2 p}} \int_{B_{r}}\left|P_{r}-P_{1}\right|^{p}\right)^{\frac{1}{p}} \leq C M(1)
$$

where we have used (3.33) with $1=\alpha r$ and $\alpha=1 / r \in(1,2]$.

Now for every $\rho \geq 1$, we write $\rho=2^{k} r$ with an integer $k \geq 1$ and $r \in\left[\frac{1}{2}, 1\right)$. Then we have

$$
\left(\frac{1}{\rho^{n+2 p}} \int_{B_{\rho}}\left|u-P_{1}\right|^{p}\right)^{\frac{1}{p}} \leq\left(\frac{1}{\rho^{n+2 p}} \int_{B_{\rho}}\left|u-P_{\rho}\right|^{p}\right)^{\frac{1}{p}}+\left(\frac{1}{\rho^{n+2 p}} \int_{B_{\rho}}\left|P_{\rho}-P_{1}\right|^{p}\right)^{\frac{1}{p}}
$$

We get

$$
\begin{aligned}
\left(\frac{1}{\rho^{n+2 p}} \int_{B_{\rho}}\left|P_{\rho}-P_{1}\right|^{p}\right)^{\frac{1}{p}} & =\left(\frac{1}{\rho^{n+2 p}} \int_{B_{\rho}}\left|P_{r}-P_{1}\right|^{p}\right)^{\frac{1}{p}}+\sum_{j=1}^{k}\left(\frac{1}{\rho^{n+2 p}} \int_{B_{\rho}}\left|P_{2^{j} r}-P_{2^{j-1} r}\right|^{p}\right)^{\frac{1}{p}} \\
& \leq C M(1)+C \sum_{j=1}^{k} M\left(2^{j} r\right)
\end{aligned}
$$

From (3.34)-(3.35), we deduce that:

$$
\begin{aligned}
\left(\frac{1}{\rho^{n+2 p}} \int_{B_{\rho}}\left|u-P_{1}\right|^{p}\right)^{\frac{1}{p}} & \leq M(\rho)+C \sum_{j=1}^{k} M\left(2^{j} r\right)+C M(1) \\
& \leq 3 C \sum_{j=1}^{k} M\left(2^{j} r\right) \\
& \leq 6 C \sum_{j=1}^{k} \frac{M\left(2^{j} r\right)}{2^{j+1} r}\left(2^{j+1} r-2^{j} r\right) \\
& \leq 6 C \int_{2 r}^{2^{k+1} r} \frac{M(s)}{s} d s \\
& \leq 6 C \int_{1}^{2 \rho} \frac{M(s)}{s} d s
\end{aligned}
$$

which ends the proof of the Lemma with $C_{1}=6 C$.

\subsection{Proof of Proposition 2.7}

We will prove the following result which will imply Proposition 2.7 because Proposition 2.5 shows that we can choose the threshold $M_{0}=+\infty$ : 
Proposition 3.2 (Modulus of continuity of the solution up to the second order) Let us assume that the set $\mathcal{P}$ is either the set of harmonic polynomials of degree less or equal to 2 , or that each element of $\mathcal{P}$ is homogeneous of degree 2.

For $p \in(1,+\infty)$, let us consider any function $u$ which satisfies

$$
\forall r \in\left(0, r_{0}\right), \quad\left(M(u, r) \leq M_{0}\right) \Longrightarrow\left(M(u, \lambda r)<\mu M(u, r) \quad \text { or } \quad M(u, r)<C_{0} \sigma_{p}(r / 2)\right)
$$

for some constants $M_{0}, C_{0}>0, r_{0}, \lambda, \mu \in(0,1)$, and a Dini modulus of continuity $\sigma_{p}$. Let us define $\alpha=\ln \mu / \ln \lambda$. If $M\left(u, r_{0}\right) \leq M_{0}$, then there exist $P_{0} \in \mathcal{P}$ and a constant $C_{0}^{\prime}>0$ only depending on $C_{0}, r_{0}, \lambda, \mu$, such that for every $\rho \in\left(0, \lambda r_{0} / 2\right)$, we have

$$
\left(\frac{1}{\rho^{n+2 p}} \int_{B_{\rho}} d y\left|u-P_{0}\right|^{p}\right)^{\frac{1}{p}} \leq C_{0}^{\prime}\left\{M\left(u, r_{0}\right) \rho^{\alpha}+\int_{0}^{\rho} \frac{\sigma_{p}(r)}{r} d r+\rho^{\alpha} \int_{\rho}^{r_{0}} \frac{\sigma_{p}(r)}{r^{1+\alpha}} d r\right\}
$$

Before to prove Proposition 3.2, we will need several Lemmata. In all what follows, we will set

$$
\sigma(r)=\sigma_{p}(r / 2)
$$

\section{Lemma 3.3 (Decay estimate of $M$ )}

Under the assumptions of Proposition 3.2, we have for every $r \in\left(0, \lambda r_{0}\right]$,

$$
M(u, r) \leq \max \left(C_{2} r^{\alpha}, \quad C_{0} r^{\alpha} \sup _{\rho \in\left[r, \lambda r_{0}\right]} \frac{\sigma(\rho)}{\rho^{\alpha}}\right)
$$

with $\alpha=\ln \mu / \ln \lambda$ and $C_{2}=M\left(u, r_{0}\right) /\left(\lambda r_{0}\right)^{\alpha}$.

\section{Proof of Lemma 3.3}

If $r \leq \lambda r_{0}$, we write it $r=\lambda^{k} r_{1}$ with an integer $k \geq 1$ and $r_{1} \in\left(\lambda r_{0}, r_{0}\right]$. Then we have

$$
\begin{aligned}
M(u, r) & \leq \max \left(C_{0} \sigma(r), \mu M(u, r / \lambda)\right) \\
& \leq \max \left(C_{0} \sigma(r), C_{0} \mu \sigma(r / \lambda), \mu^{2} M\left(u, r / \lambda^{2}\right)\right) \\
& \leq \max \left(C_{0} \sigma(r), C_{0} \mu \sigma(r / \lambda), C_{0} \mu^{2} \sigma\left(r / \lambda^{2}\right), \ldots, C_{0} \mu^{k-1} \sigma\left(r / \lambda^{k-1}\right), \mu^{k} M\left(u, r / \lambda^{k}\right)\right)
\end{aligned}
$$

Now for $\rho=r / \lambda^{j}$ with $j \geq 1$, we have on the one hand

$$
\begin{aligned}
\mu^{j} \sigma\left(r / \lambda^{j}\right) & =\sigma(\rho) e^{j \ln \mu} \\
& =\sigma(\rho) e^{\ln (r / \rho) \frac{\ln \mu}{\ln \lambda}} \\
& =\frac{\sigma(\rho)}{\rho^{\alpha}} r^{\alpha}
\end{aligned}
$$


where $\alpha=\ln \mu / \ln \lambda$. On the other hand, we have

$$
\begin{aligned}
\mu^{k} M\left(u, r / \lambda^{k}\right) & \leq \mu^{k} M\left(u, r_{1}\right) \\
& \leq \mu^{k} M\left(u, r_{0}\right) \\
& =C_{2} \mu^{k}\left(\lambda r_{0}\right)^{\alpha} \\
& \leq C_{2} \mu^{k} r_{1}^{\alpha} \\
& =C_{2} \mu^{k}\left(\frac{r}{\lambda^{k}}\right)^{\alpha} \\
& =C_{2} r^{\alpha}
\end{aligned}
$$

Therefore we deduce that

$$
M(u, r) \leq \max \left(C_{0} r^{\alpha} \sup _{\rho \in\left[r, \lambda r_{0}\right]} \frac{\sigma(\rho)}{\rho^{\alpha}}, \quad C_{2} r^{\alpha}\right)
$$

which ends the proof of the Lemma.

\section{Lemma 3.4 (Decay estimate)}

Under the assumptions of Proposition 3.2, there exists $C=C\left(\lambda, \mu, C_{0}\right)>0$ such that for $R \leq r_{0}$ we have

$$
\int_{0}^{\lambda R} \frac{M(u, r)}{r} d r \leq M\left(u, r_{0}\right) \frac{1}{\alpha}\left(\frac{R}{r_{0}}\right)^{\alpha}+C\left(\int_{0}^{\lambda R} \frac{\sigma(r)}{r} d r+R^{\alpha} \int_{\lambda R}^{r_{0}} \frac{\sigma(r)}{r^{1+\alpha}} d r\right)
$$

with $\alpha=\ln \mu / \ln \lambda$.

\section{Proof of Lemma 3.4}

We first remark that for $r \leq \lambda r_{0}$ we have

$$
\begin{aligned}
\sup _{\rho \in\left[r, \lambda r_{0}\right]} \frac{\sigma(\rho)}{\rho^{\alpha}} & =\frac{\sigma\left(\rho_{0}\right)}{\rho_{0}^{\alpha}} & & \text { for some } \rho_{0} \in\left[r, \lambda r_{0}\right] \\
& \leq \frac{1}{\rho_{0}^{\alpha}} \frac{1}{t \rho_{0}} \int_{\rho_{0}}^{\rho_{0}+t \rho_{0}} \sigma(\rho) d \rho & & \text { with } \quad t=\frac{1-\lambda}{\lambda}>0 \\
& \leq \frac{1}{t \lambda^{1+\alpha}} \int_{\rho_{0}}^{\frac{\rho_{0}}{\lambda}} \frac{\sigma(\rho)}{\rho^{1+\alpha}} d \rho & & \text { with } \quad t=\frac{1-\lambda}{\lambda}>0 \\
& \leq C_{3} \int_{r}^{r_{0}} \frac{\sigma(\rho)}{\rho^{1+\alpha}} d \rho & & \text { with } \quad C_{3}=\frac{1}{(1-\lambda) \lambda^{\alpha}}>0
\end{aligned}
$$

We deduce that

$$
\int_{0}^{\lambda R} \frac{M(u, r)}{r} d r \leq C_{2} \int_{0}^{\lambda R} r^{\alpha-1} d r+C_{0} C_{3} J
$$


with

$$
\begin{aligned}
J & :=\int_{0}^{\lambda R} d r r^{\alpha-1}\left(\int_{r}^{r_{0}} \frac{\sigma(\rho)}{\rho^{1+\alpha}} d \rho\right) \\
& =\int_{0}^{\lambda R} d r \frac{r^{\alpha}}{\alpha} \frac{\sigma(r)}{r^{1+\alpha}} d r+[A(r)]_{0}^{\lambda R} \text { with } A(r)=\frac{r^{\alpha}}{\alpha}\left(\int_{r}^{r_{0}} \frac{\sigma(\rho)}{\rho^{1+\alpha}} d \rho\right) \\
& =\frac{1}{\alpha} \int_{0}^{\lambda R} \frac{\sigma(r)}{r} d r+A(\lambda R)
\end{aligned}
$$

where for the second line we have used integration by parts, and for the third line the fact that $A\left(0^{+}\right)=0$, comming from the dominated convergence theorem applied to

$$
\alpha A(r)=\int_{0}^{r_{0}} h_{r}(\rho)\left(\frac{\sigma(\rho)}{\rho}\right) d \rho \quad \text { with } \quad h_{r}(\rho):=1_{\{\rho \geq r\}}\left(\frac{r}{\rho}\right)^{\alpha}
$$

with $0 \leq h_{r}(\rho) \leq 1$ and $h_{r}(\rho) \longrightarrow 0$ for a.e. $\rho \in\left[0, r_{0}\right]$ as $r \longrightarrow 0$.

We get the result with $C=C_{0} C_{3} / \alpha$.

Lemma 3.5 (Modulus of continuity of the solution up to the second order)

If $u$ is defined on $B_{r_{0}}$, then there exists $P_{0} \in \mathcal{P}$ such that for every $\rho \in\left(0, r_{0} / 2\right)$, we have

$$
\left(\frac{1}{\rho^{n+2 p}} \int_{B_{\rho}} d y\left|u-P_{0}\right|^{p}\right)^{\frac{1}{p}} \leq C_{1} \int_{0}^{2 \rho} \frac{M(u, r)}{r} d r
$$

\section{Proof of Lemma 3.5}

We assume that $u$ is defined on $B_{r_{0}}$. From Lemma 2.9 applied to $u^{r}(x)=u(r x) / r^{2}$, we get

$$
N\left(u_{r}, 1\right)=\left(\int_{B_{1}}\left|u^{r}-P^{r}\right|^{p}\right)^{\frac{1}{p}} \quad \text { for some } \quad P^{r} \in \mathcal{P}
$$

and for $2 \gamma r \leq r_{0}$ with $\gamma \geq 1$, we have

$$
\left(\frac{1}{\gamma^{n+2 p}} \int_{B_{\gamma}(0)}\left|u^{r}-P^{r}\right|^{p}\right)^{\frac{1}{p}} \leq C_{1} \int_{1}^{2 \gamma} \frac{M\left(u^{r}, s\right)}{s} d s
$$

A change of variables with $\rho=\gamma r$ and $P^{r}(x)=P_{r}(r x) / r^{2}$ allows to see that (using $M\left(u^{r}, s\right)=$ $M(u, r s))$

$$
\left(\frac{1}{\rho^{n+2 p}} \int_{B_{\rho}}\left|u-P_{r}\right|^{p}\right)^{\frac{1}{p}} \leq C_{1} \int_{r}^{2 \rho} \frac{M(u, t)}{t} d t
$$

Now for $\rho \in\left(0, r_{0} / 2\right)$ fixed, we can pass to the limit as $r$ goes to zero, and up to extraction of a subsequence we can assume that $P_{r} \longrightarrow P_{0} \in \mathcal{P}$, and we get

$$
\left(\frac{1}{\rho^{n+2 p}} \int_{B_{\rho}}\left|u-P_{0}\right|^{p}\right)^{\frac{1}{p}} \leq C_{1} \int_{0}^{2 \rho} \frac{M(u, t)}{t} d t
$$


This ends the proof.

We are now ready to prove Proposition 3.2.

\section{Proof of Proposition 3.2}

Just apply Lemma 3.5 and Lemma 3.4. We get

$$
\left(\frac{1}{\rho^{n+2 p}} \int_{B_{\rho}} d y\left|u-P_{0}\right|^{p}\right)^{\frac{1}{p}} \leq C_{1}\left\{M\left(u, r_{0}\right) \frac{1}{\alpha}\left(\frac{2 \rho}{\lambda r_{0}}\right)^{\alpha}+C\left(\int_{0}^{2 \rho} \frac{\sigma(r)}{r} d r+\left(\frac{2 \rho}{\lambda}\right)^{\alpha} \int_{2 \rho}^{r_{0}} \frac{\sigma(r)}{r^{1+\alpha}} d r\right)\right\}
$$

which implies the result with $\sigma(r)=\sigma_{p}(r / 2)$. This ends the proof of the Proposition.

\section{General results for the obstacle problem: proof of Proposition 1.5 and of Theorem 1.6}

We recall that we are interested in solution $u$ of (1.8), that we recall for the convenience of the reader for $p \in(\max (n / 2,1),+\infty)$

$$
\left\{\begin{array}{l}
\Delta u=f(x) \cdot 1_{\{u>0\}} \mid \text { on } B_{1} \\
u \geq 0 \\
f \in L^{p}\left(B_{1}\right) \text { and } f(0)=1 \\
0 \in \partial\{u>0\}
\end{array}\right.
$$

In this section we will prove estimates on the pointwise quadratic growth and on the classification in degenerate/regular/singular points using the "monotonicity formula".

\subsection{Quadratic growth of the solution}

\section{Proof of Proposition 1.5}

For simplicity, we fix the exponent $p$, and set

$$
\sigma(r)=\sigma_{p}(r)
$$

By the way, we will have to consider sequences of modulus of continuity $\sigma$ that we denote by $\left(\sigma_{m}\right)_{m}$ indexed by $m$, with no possible confusion.

Let us first remark that defining

$$
g=f \cdot 1_{\{u>0\}}
$$

we have with obvious notation for the corresponding modulus of continuity

$$
\sigma^{g} \leq \sigma^{f}+|f(0)|
$$

therefore we can apply Proposition 2.3 with finite modulus of continuity $\sigma^{g}$, and conclude that

$$
\forall r \in(0,1), \quad \exists P_{r} \in \mathcal{P}_{2}, \quad\left(\frac{1}{\left|B_{r}\right|} \int_{B_{r}}\left|\frac{u-P_{r}}{r^{2}}\right|^{p}\right)^{\frac{1}{p}} \leq C\left\{\left(\int_{B_{1}}|u|^{p}\right)^{\frac{1}{p}}+\sigma(1)+1\right\}
$$


with

$$
\mathcal{P}_{2}=\left\{P(x)=a+b \cdot x+\frac{1}{2} t x \cdot c \cdot x, \quad(a, b, c) \in \mathbb{R} \times \mathbb{R}^{n} \times \mathbb{R}_{s y m}^{n \times n}, \quad \text { trace }(c)=0\right\}
$$

Let us write

$$
P_{r}(x)=a_{r}+b_{r} \cdot x+\frac{1}{2} t x \cdot c_{r} \cdot x
$$

Step 1 : estimate on $a_{r} / r^{2}$

Let us now remark that

$$
\Delta u=g \quad \text { on } \quad B_{1}
$$

and then by the classical $W^{2, p}$ elliptic estimates and the Sobolev imbbedings, we get that there exists a constant $C_{1}>0$ such that for every $x, y \in B_{1 / 2}$ :

$$
\begin{aligned}
|u(x)-u(y)| & \leq C_{1}|x-y|^{\alpha}\left\{\left(\frac{1}{\left|B_{1}\right|} \int_{B_{1}}|u|^{p}\right)^{\frac{1}{p}}+\left(\frac{1}{\left|B_{1}\right|} \int_{B_{1}}|g|^{p}\right)^{\frac{1}{p}}\right\} \\
& \leq C_{1}|x-y|^{\alpha}\left\{\left(\frac{1}{\left|B_{1}\right|} \int_{B_{1}}|u|^{p}\right)^{\frac{1}{p}}+\left(\frac{1}{\left|B_{1}\right|} \int_{B_{1}}|f(x)-f(0)|^{p}\right)^{\frac{1}{p}}+|f(0)|\right\}
\end{aligned}
$$

for $\alpha=\min \left(1,2-\frac{n}{p}\right)$. Let us now set for $P_{r}$ realizing the infimum in the Definition 2.2 of $N(u, r)$

$$
w_{r}(x)=\frac{\left(u-P_{r}\right)(r x)}{r^{2}}
$$

Applying the previous result to $w_{r}$ (and a rescaling in the ball $B_{r}$ ), we get that for every $x, y \in B_{1 / 2}$, we have

$$
\left|w_{r}(x)-w_{r}(y)\right| \leq C|x-y|^{\alpha}\left\{N_{2}(u, r)+\left(\frac{1}{\left|B_{r}\right|} \int_{B_{r}}|f(x)-f(0)|^{p}\right)^{\frac{1}{p}}+|f(0)|\right\}
$$

We then write (using the fact that $u(0)=0$, and then $\left.-\frac{a_{r}}{r^{2}}=w_{r}(0)=w_{r}(x)-\left(w_{r}(x)-w_{r}(0)\right)\right)$ for $\rho \in(0,1 / 2)$

$$
\begin{aligned}
\frac{\left|a_{r}\right|}{r^{2}} & \leq\left(\frac{1}{\left|B_{\rho}\right|} \int_{B_{\rho}}\left|w_{r}-w_{r}(0)\right|^{p}\right)^{\frac{1}{p}}+\left(\frac{1}{\left|B_{\rho}\right|} \int_{B_{\rho}}\left|w_{r}\right|^{p}\right)^{\frac{1}{p}} \\
& \leq C \rho^{\alpha}\left\{N_{2}(u, r)+\sigma(r)+1\right\}+\left|B_{\rho}\right|^{-\frac{1}{p}} N_{2}(u, r)
\end{aligned}
$$

Using the bound given by Proposition 2.3 on $M_{2}(u, r) \geq N_{2}(u, r)$, we deduce that there exists a constant $C_{0}>0$ such that

$$
\frac{\left|a_{r}\right|}{r^{2}} \leq C_{0}\left\{\left(\int_{B_{1}}|u|^{p}\right)^{\frac{1}{p}}+\sigma(1)+1\right\}
$$


Step 2 : estimate on $\left|b_{r}\right| / r+\left|c_{r}\right|$

We want to prove that there exists a constant $C_{0}^{\prime}>0$ such that

$$
\frac{\left|b_{r}\right|}{r}+\left|c_{r}\right|<C_{0}^{\prime}\left\{\left(\int_{B_{1}}|u|^{p}\right)^{\frac{1}{p}}+\sigma(1)+1\right\}
$$

If it is false, then there exist sequences $\left(r_{m}\right)_{m},\left(C_{m}\right)_{m},\left(u_{m}\right)_{m},\left(f_{m}\right)_{m},\left(\sigma_{m}\right)_{m}$ such that

$$
r_{m} \longrightarrow 0 \text { and } C_{m} \longrightarrow+\infty
$$

and

$$
M_{m}:=\frac{\left|b_{r_{m}}\right|}{r_{m}}+\left|c_{r_{m}}\right| \geq C_{m}\left\{\left(\int_{B_{1}}\left|u_{m}\right|^{p}\right)^{\frac{1}{p}}+\sigma_{m}(1)+1\right\}
$$

Let us define

$$
v_{m}(x)=\frac{u_{m}\left(r_{m} x\right)}{r_{m}^{2} M_{m}} \geq 0 \quad \text { and } \quad P_{m}(x)=\frac{P_{r_{m}}\left(r_{m} x\right)}{r_{m}^{2} M_{m}}
$$

Then we have (from (4.37))

$$
\left(\frac{1}{\left|B_{1}\right|} \int_{B_{1}}\left|v_{m}-P_{m}\right|^{p}\right)^{\frac{1}{p}} \leq \frac{C}{C_{m}} \longrightarrow 0
$$

and

$$
\frac{\left|a_{r_{m}}\right|}{r_{m}^{2} M_{m}} \leq \frac{C_{0}}{C_{m}} \longrightarrow 0
$$

We deduce, up to extract a subsequence, that $P_{m}$ converges to a harmonic polynomial $P_{\infty}$ which can be written

$$
P_{\infty}(x)=b_{\infty} \cdot x+\frac{1}{2} t x \cdot c_{\infty} \cdot x \quad \text { with } \quad\left|b_{\infty}\right|+\left|c_{\infty}\right|=1
$$

By contruction $v_{m} \geq 0$ also converges to $P_{\infty}$ on $B_{1}$, and then $P_{\infty} \geq 0$ on $B_{1}$. Therefore $P_{\infty}=0$. Contradiction.

\section{Step 3 : Conclusion}

Therefore we conclude that there exists a constant $C_{0}^{\prime \prime}>0$ such that

$$
\sup _{B_{r}} \frac{\left|P_{r}\right|}{r^{2}} \leq C_{0}^{\prime \prime}\left\{\left(\int_{B_{1}}|u|^{p}\right)^{\frac{1}{p}}+\sigma(1)+1\right\}
$$

and then there exists $C_{3}>0$ such that

$$
\forall r \in(0,1), \quad\left(\frac{1}{\left|B_{r}\right|} \int_{B_{r}}\left|\frac{u}{r^{2}}\right|^{p}\right)^{\frac{1}{p}} \leq C_{3}\left\{\left(\int_{B_{1}}|u|^{p}\right)^{\frac{1}{p}}+\sigma(1)+1\right\}
$$

Then applying again interior $W^{2, p}$ elliptic estimates and Sobolev imbeddings, we get that there exists a constant $C_{4}>0$ such that

$$
\forall x \in B_{1 / 2}, \quad|u(x)| \leq|x|^{2} C_{4}\left\{\left(\int_{B_{1}}|u|^{p}\right)^{\frac{1}{p}}+\sigma(1)+1\right\}
$$

This ends the proof of the Proposition. 


\section{Corollary 4.1 (Bound on $U$ )}

Let $p \in(\max (n / 2,1),+\infty)$ with $p \geq 2 n /(n+1)$. Then there exists constants $C>0$ such that if $u$ is a solution of (1.8) with $\sigma_{p}$ given by (1.9), then $U=x \cdot \nabla u-2 u$ satisfies for $\frac{1}{p}+\frac{1}{p^{\prime}}=1$

$$
\forall r \in(0,1 / 2), \quad\left(\frac{1}{B_{r}} \int_{B_{r}}\left|\frac{U}{r^{2}}\right|^{p^{\prime}}\right)^{\frac{1}{p^{\prime}}} \leq C\left\{\left(\int_{B_{1}}|u|^{p}\right)^{\frac{1}{p}}+\sigma_{p}(1)+1\right\}
$$

\section{Proof of Corollary 4.1}

Let us define

$$
u_{r}(x)=\frac{u(r x)}{r^{2}}, \quad f_{r}(x)=f(r x)
$$

We have $\Delta u_{r}=f_{r} \cdot 1_{\left\{u_{r}>0\right\}}$. From classical interior $W^{2, p}$ elliptic estimates and Sobolev imbbedings applied to $u_{r}$, we get for $r \in(0,1 / 2)$ the existence of a constant $C>0$ such that (because $p \geq 2 n /(n+1)$ )

$$
\left(\frac{1}{B_{1}} \int_{B_{1}}\left|u_{r}\right|^{p^{\prime}}\right)^{\frac{1}{p^{\prime}}}+\left(\frac{1}{B_{1}} \int_{B_{1}}\left|\nabla u_{r}\right|^{p^{\prime}}\right)^{\frac{1}{p^{\prime}}} \leq C\left\{\left(\frac{1}{\left|B_{1}\right|} \int_{B_{1}}\left|u_{r}\right|^{p}\right)^{\frac{1}{p}}+\sigma_{p}(r)+1\right\}
$$

Therefore

$$
\begin{aligned}
\left(\frac{1}{B_{1}} \int_{B_{1}}\left|x \cdot \nabla u_{r}-2 u_{r}\right|^{p^{\prime}}\right)^{\frac{1}{p^{\prime}}} & \leq 3 C\left\{\left(\frac{1}{\left|B_{1}\right|} \int_{B_{1}}\left|u_{r}\right|^{p}\right)^{\frac{1}{p}}+\sigma_{p}(r)+1\right\} \\
& \leq C_{2}\left\{\left(\frac{1}{\left|B_{1}\right|} \int_{B_{1}}|u|^{p}\right)^{\frac{1}{p}}+\sigma_{p}(1)+1\right\}
\end{aligned}
$$

for some constant $C_{2}>0$ where we have used Proposition 1.5 for the last line. This is exactly the expected result for $U=x \cdot u-2 u$, which ends the proof of the Corollary.

\subsection{Liouville Theorem and monotonicity formula}

The following result, proved by Caffarelli [7] and Weiss [38], classifies the possible blow-up limits:

\section{Theorem 4.2 (Liouville theorem)}

Let us consider a function $u^{0}$ which is solution of

$$
\left\{\begin{array}{l}
\Delta u^{0}=1_{\left\{u^{0}>0\right\}} \quad \text { on } \quad \mathbb{R}^{n} \\
u^{0} \geq 0 \\
u^{0}(\lambda x)=\lambda^{2} u^{0}(x) \quad \text { for any } \quad \lambda>0
\end{array}\right.
$$


Then either

i) (degenerate case) $u^{0}(x) \equiv 0$, or

ii) (regular case) there exists $\nu \in \mathbf{S}^{n-1}$ such that

$$
u^{0}(x)=\frac{1}{2} \max (0, x \cdot \nu)^{2}
$$

or

iii) (singular case) there exists a symmetric matrix $Q \in \mathbb{R}_{\text {sym }}^{n \times n}$ with $Q \geq 0$, $\operatorname{trace}(Q)=1$ such that

$$
u^{0}(x)=\frac{1}{2} t x \cdot Q \cdot x
$$

Sketch of the proof of Theorem 1.6

It is possible to compute (see [32]):

$$
\frac{d \Phi}{d r}(r)=\frac{2}{r^{n}} \int_{\partial B_{r}}\left|\frac{U(x)}{r^{2}}\right|^{2}-\frac{2}{r^{n+3}} \int_{B_{r}} U(f(x)-f(0))
$$

where $r=|x|$ in the integral on the boundary $\partial B_{r}$, and

$$
U=x \cdot \nabla u-2 u
$$

It can be easily checked that this computation is justified for $p \geq 2 n /(n+1)$ which garanties in particular that $\nabla u \in L^{2}\left(\partial B_{r}\right)$ and $\nabla u \cdot \nabla U \in L_{l o c}^{1}\left(B_{1}\right)$. We have

$$
\frac{1}{\left|B_{r}\right| r^{3}} \int_{B_{r}}|U(f(x)-f(0))| \leq\left(\frac{1}{\left|B_{r}\right|} \int_{B_{r}}\left|\frac{U(x)}{r^{2}}\right|^{p^{\prime}}\right)^{\frac{1}{p^{\prime}}} \cdot \frac{\sigma_{p}(r)}{r}
$$

The bound of Corollary 4.1 implies that there exists a constant $C>0$ such that

$$
\frac{2}{r^{n+3}} \int_{B_{r}}|U(f(x)-f(0))| \leq C \frac{\sigma_{p}(r)}{r}
$$

and then for $r>t>0$

$$
\Phi(r)-\Phi(t) \geq-C \int_{t}^{r} \frac{\sigma_{p}(s)}{s} d s
$$

This implies that $\Phi(r)$ has a limit $\Phi\left(0^{+}\right)$in $r=0$.

The rest of the proof is similar to what is done in the constant coefficient case (see [38] and [7]). This ends the proof of the Theorem.

\section{Degenerate points of the free boundary}

We first start with the following result

\section{Lemma 5.1 (Nondegeneracy)}

Let $p \in(\max (n / 2,1),+\infty)$. Then there exists a constant $C_{0}>0$ such that if $u$ satisfies for some $R>0$ :

$$
\left\{\begin{array}{l}
\Delta u=f \cdot 1_{\{u>0\}} \quad \text { on } \quad B_{R} \\
u \geq 0
\end{array}\right.
$$


and if $B_{d}\left(x_{0}\right) \subset B_{R}$ for some $d>0$, then for

$$
\lambda:=C_{0} d^{2-\frac{n}{p}} R^{\frac{n}{p}}\left(\frac{1}{\left|B_{R}\right|} \int_{B_{R}}|f(x)-f(0)|^{p}\right)^{\frac{1}{p}}
$$

we have

$$
\left(u\left(x_{0}\right)>2 \lambda\right) \quad \Longrightarrow \quad\left(\sup _{\partial B_{d}\left(x_{0}\right)} u \geq \frac{d^{2}}{2 n}-2 \lambda\right)
$$

\section{Proof of Lemma 5.1}

Let us consider the solution $v$ of

$$
\begin{cases}\Delta v=f(x)-f(0) & \text { on } \quad B_{d}\left(x_{0}\right) \\ v=0 & \text { on } \quad \partial B_{d}\left(x_{0}\right)\end{cases}
$$

From the classical $W^{2, p}$ elliptic estimates and the Sobolev imbbedings for $p>n / 2$, we deduce that there exists a constant $C_{0}>0$ such that (using moreover a rescaling argument comming back to the unit ball)

$$
|v|_{L^{\infty}\left(B_{d}\left(x_{0}\right)\right)} \leq C_{0} d^{2}\left(\frac{1}{\left|B_{d}\right|} \int_{B_{d}\left(x_{0}\right)}|f(x)-f(0)|^{p}\right)^{\frac{1}{p}} \leq \lambda
$$

We now define

$$
w(x)=u(x)-\frac{\left|x-x_{0}\right|^{2}}{2 n}-v(x)
$$

which satisfies

$$
\Delta w=0 \quad \text { on } \quad \omega:=\{u>0\} \cap B_{d}\left(x_{0}\right)
$$

then from the maximum principle, we have

$$
w\left(x_{0}\right) \leq \sup _{\partial \omega} w
$$

But on the one hand, we have

$$
w\left(x_{0}\right)=u\left(x_{0}\right)-v\left(x_{0}\right) \geq u\left(x_{0}\right)-|v|_{L^{\infty}\left(B_{d}\left(x_{0}\right)\right)} \geq u\left(x_{0}\right)-\lambda
$$

and

$$
w(x)=-\frac{\left|x-x_{0}\right|^{2}}{2 n}-v(x)<\lambda \quad \text { on } \quad(\partial\{u>0\}) \cap B_{d}\left(x_{0}\right)
$$

Therefore, while $u\left(x_{0}\right)>2 \lambda$, we get that

$$
\sup _{\partial \omega} w=\sup _{\{u>0\} \cap \partial B_{d}\left(x_{0}\right)} w \leq \sup _{\partial B_{d}\left(x_{0}\right)} u-\frac{d^{2}}{2 n}+|v|_{L^{\infty}\left(B_{d}\left(x_{0}\right)\right)}
$$

and therefore

$$
\sup _{\partial B_{d}\left(x_{0}\right)} u \geq \frac{d^{2}}{2 n}-2|v|_{L^{\infty}\left(B_{d}\left(x_{0}\right)\right)}
$$

which ends the proof of the Lemma. 


\section{Proposition 5.2 (Growth at degenerate a point)}

Let $p \in(\max (n / 2,1),+\infty)$ with $p \geq 2 n /(n+1)$. There exists a constant $C>0$ such that if 0 is a degenerate point for $u$, then there exists $r_{0}$ (here depending on $u$ ) such that

$$
\forall x \in B_{r_{0}}, \quad 0 \leq u(x) \leq C|x|^{2} \sigma_{p}(2|x|)
$$

\section{Proof of Proposition $\mathbf{5 . 2}$}

Assume that the Proposition is false. Then there exists sequences $\left(C_{m}\right)_{m},\left(x_{m}\right)_{m}$ such that

$$
C_{m} \longrightarrow+\infty \text { and } x_{m} \longrightarrow 0
$$

and

$$
u\left(x_{m}\right) \geq C_{m}\left|x_{m}\right|^{2} \sigma_{p}\left(2\left|x_{m}\right|\right)
$$

Apply Lemma 5.1 with $\left|x_{m}\right|=d_{m}=R_{m} / 2$. Then we get that there exists a constant $C_{1}>0$ such that

$$
\sup _{\partial B_{d_{m}}\left(x_{m}\right)} u \geq \frac{d_{m}^{2}}{2 n}-C_{1} d_{m}^{2} \sigma_{p}\left(2 d_{m}\right)
$$

and then

$$
\sup _{\partial B_{R_{m}}} u \geq \frac{R_{m}^{2}}{8 n}-\frac{C_{1}}{4} R_{m}^{2} \sigma_{p}\left(R_{m}\right)
$$

Then we see that up to extraction of a subsequence, we have

$$
u_{m}(x)=\frac{u\left(R_{m} x\right)}{R_{m}^{2}} \longrightarrow u_{\infty}(x) \not \equiv 0
$$

On the other hand, if we note $\Phi_{u}(r)$ the expression (1.10) associated to the function $u$, we get

$$
\Phi_{u}\left(r R_{m}\right) \longrightarrow \Phi\left(0^{+}\right)=0
$$

and

$$
\Phi_{u}\left(r R_{m}\right)=\Phi_{u_{m}}(r) \longrightarrow \Phi_{u_{\infty}}(r)
$$

which implies that $u_{\infty} \equiv 0$. Contradiction. This ends the proof of the Proposotion.

\section{Example of a degenerate point}

For $p>(\max (n / 2,1),+\infty)$, we build here a function $f \in L^{p}\left(B_{1}\right)$ with $\sigma_{p}$ Dini such that $u$ solves on $B_{1}$

$$
\Delta u=f \cdot 1_{\{u>0\}} \quad \text { and } \quad u \geq 0
$$

and the origin 0 is a degenerate point for $u$ (see Figure 1 ).

Let $\zeta \in C_{c}^{\infty}\left(\mathbb{R}^{n}\right)$ such that $\operatorname{supp} \zeta \subset B_{1 / 4}, \zeta \geq 0$ and $\zeta \not \equiv 0$. We set $\gamma=1+|\Delta \zeta|_{L^{\infty}\left(\mathbb{R}^{n}\right)}$ and for $\lambda>0$

$$
\zeta_{\lambda}(x)=\lambda^{2} \zeta\left(\frac{x}{\lambda}\right)
$$

We also set $x=\left(x_{1}, x^{\prime}\right)$ with $x^{\prime}=\left(x_{2}, \ldots, x_{n}\right)$. Given a non-increasing sequence $\left(\lambda_{k}\right)_{k}$ with $0<\lambda_{k} \leq 1$, we set

$$
u(x)=\sum_{k \geq 1} 4^{\frac{n}{p}} \zeta_{\lambda_{k} 2^{-k}}\left(x_{1}-2^{-k}, x^{\prime}\right)
$$


and define for $x^{k}=\left(2^{-k}, 0, \ldots, 0\right)$

$$
\Omega=\bigcup_{k \geq 1} B_{\lambda_{k} 2^{-k}}\left(x^{k}\right)
$$

Let us define with $f(0)=1$

$$
f=\left\{\begin{array}{lll}
\Delta u & \text { on } & B_{1} \cap \Omega \\
1 & \text { on } & B_{1} \backslash \Omega
\end{array}\right.
$$

the union of disjoint balls. Then we compute for $K \geq 1$

$$
\begin{aligned}
& \frac{1}{\left|B_{2^{-K}}\right|} \int_{B_{2}-K}|f(x)-f(0)|^{p} \\
& \leq \frac{1}{\left|B_{2^{-K}}\right|} \sum_{k \geq K} 4^{n} \gamma^{p}\left(\lambda_{k} 2^{-k}\right)^{n}\left|B_{1 / 4}\right| \\
& \leq \gamma^{p} \lambda_{K}^{n} 2^{n K} \sum_{k \geq K} 2^{-n k} \\
& \leq \mu^{p} \lambda_{K}^{n} \quad \text { with } \quad \mu^{p}=\gamma^{p}\left(1-2^{-n}\right)^{-1}
\end{aligned}
$$

Therefore

$$
\left(\frac{1}{\left|B_{2^{-K}}\right|} \int_{B_{2^{-K}}}|f(x)-f(0)|^{p}\right)^{\frac{1}{p}} \leq \mu \lambda_{K}^{\frac{n}{p}}
$$

Hence $\sigma_{p}$ is Dini, if we choose the sequence $\left(\lambda_{k}\right)_{k}$ such that

$$
\sum_{K \geq 1} \lambda_{K}^{\frac{n}{p}}<+\infty
$$

(for instance for a geometric sequence $\left.\left(\lambda_{K}\right)_{K}\right)$.

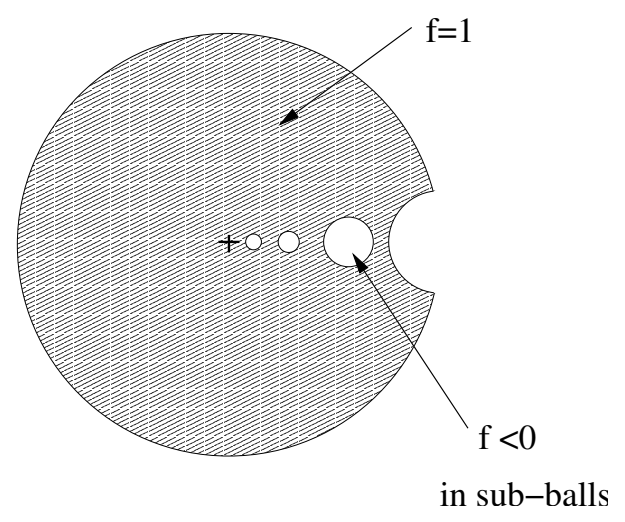

Figure 1: Construction of a function $f$ such that the origin is a degenerate point 


\section{Regular points of the free boundary and proof of Theorem 1.8}

\subsection{Proof of Theorem 1.8}

Here we adapt the proof of Theorem 2.1, and replace the set $\mathcal{P}_{2}$ by

$$
\mathcal{P}_{\text {reg }}=\left\{P, \quad \exists \nu \in \mathbf{S}^{n-1}, \quad P=\frac{1}{2} \max (0, x \cdot \nu)^{2}\right\}
$$

keeping the same notation for $M(u, \rho)$ and $N(u, r)$ (see Definition 2.2). Here the set $\mathcal{P}_{\text {reg }}$ contains the possible limit behaviours of the solution at the origin when the origin is a regular point.

We claim the following

\section{Proposition 6.1 (Finiteness of $M$ )}

Let us assume that $p \in(\max (n / 2,1),+\infty)$. Then there exists a constant $C>0$ such that

$$
\forall r \in(0,1), \quad M(u, r) \leq C\left\{\left(\int_{B_{1}}|u|^{p}\right)^{\frac{1}{p}}+\sigma_{p}(1)+1\right\}
$$

\section{Proof of Proposition 6.1}

This is a straightforward consequence of Proposition 1.5.

Then we have the following cornerstone result which will be proved in subsection 6.3.

\section{Proposition 6.2 (Decay estimate in a smaller ball)}

Given $p \in(n / 2,+\infty)$, there exist constants $M_{0}, C_{0}>0, r_{0}, \lambda, \mu \in(0,1)$ (only depending on $p$ and the dimension $n$ ) such that for every functions $u$ and $f$ satisfying (1.8) with a modulus of continuity $\sigma_{p}$ given by (1.9), then we have the following property

$$
\forall r \in\left(0, r_{0}\right), \quad\left(M(u, r) \leq M_{0}\right) \Longrightarrow\left(M(u, \lambda r)<\mu M(u, r) \quad \text { or } \quad M(u, r)<C_{0} \sigma_{p}(r / 2)\right)
$$

Remark 6.3 Because the problem is nonlinear here, so $M(u, r)$ has to be small enough (less than the threshold $M_{0}$ ) to satisfy the decay estimate. Just think to the case of blow-up limits $u^{0}$ at singular points, like for instance $u^{0}(x)=\frac{x^{2}}{2 n}$ for which we have $M\left(u^{0}, r\right)>M_{0}$.

\section{Proof of Theorem 1.8}

The proof of Theorem 1.8 follows exactly the lines of the proof of Theorem 2.1, where Proposition 2.7 is replaced by Proposition 3.2. This ends the proof of the Theorem.

\subsection{Preliminary results}

To prove Proposition 6.2, we will need the following Caccioppoli estimate for the obstacle problem. 


\section{Lemma 6.4 (Caccioppoli type estimate)}

Lemma 2.8 is still valid for solutions $u$ of (1.8) for P satisfying

$$
-\Delta P+1_{\{P>0\}}=0 \quad \text { and } \quad P \geq 0
$$

\section{Proof of Lemma 6.4}

On the ball $B_{1}$, we have

$$
-\Delta u+f(x) \cdot 1_{\{u>0\}}=0
$$

and let us consider any $P$ satisfying

$$
-\Delta P+1_{\{P>0\}}=0
$$

Taking the difference of these two equations, and multiplying by $\zeta^{2} w|w|^{p-2}$ for $w=u-P$, we get

$$
\int_{\mathbb{R}^{n}}-\zeta^{2} w|w|^{p-2} \Delta w+\zeta^{2} w|w|^{p-2}\left(1_{\{u>0\}}-1_{\{P>0\}}\right)=\int_{B_{R}}-\zeta^{2} w|w|^{p-2}(f(x)-f(0)) 1_{\{u>0\}}
$$

The rest of the proof is identical to the one of Lemma 2.8, simply taking into account the fact that $w\left(1_{\{u>0\}}-1_{\{P>0\}}\right) \geq 0$. This ends the proof of the Lemma.

We will also use the following result

\section{Lemma 6.5 (Weak nondegeneracy)}

Let us fix $R>0$ and $p \in(\max (n / 2,1),+\infty)$. Let us consider sequences of functions $\left(u_{m}\right)_{m}$, $\left(f_{m}\right)_{m}$ such that

$$
\left\{\begin{array}{l}
\Delta u_{m}=f_{m}(x) \cdot 1_{\left\{u_{m}>0\right\}} \mid \quad \text { on } B_{R} \\
u_{m} \geq 0 \\
f_{m}(0)=1, \quad \text { and }\left(\frac{1}{\left|B_{R}\right|} \int_{B_{R}}\left|f_{m}(x)-f_{m}(0)\right|^{p}\right)^{\frac{1}{p}}=: \tau_{m} \longrightarrow 0 \quad \text { as } \quad m \longrightarrow+\infty
\end{array}\right.
$$

Let us assume that $u_{m}$ converges to $u_{\infty}$ in $L_{\text {loc }}^{\infty}\left(B_{R}\right)$. Let us consider a compact $K$ contained in the interior of the coincidence set $\left\{u_{\infty}=0\right\}$. Then there exists a constant $C>0$ (which depends in particular on $K$ and $R$, but is independent on $\tau_{m}$ ) such that

$$
u_{m} \leq C \tau_{m} \quad \text { on } \quad K
$$

\section{Proof of Lemma 6.5}

Let us assume that the Lemma is false. Then we can find a sequence of points $\left(x_{m}\right)_{m}$ a sequence $\left(C_{m}\right)_{m}$ such that (up to extraction)

$$
\begin{gathered}
C_{m} \longrightarrow+\infty \\
u_{m}\left(x_{m}\right)>C_{m} \tau_{m}, \quad x_{m} \in K
\end{gathered}
$$

Let us choose $d$ such that $0<d<\operatorname{dist}\left(K,\left\{u_{\infty}>0\right\}\right)$. Then we can apply Lemma 5.1 which states that

$$
\left(u_{m}\left(x_{m}\right)>2 \lambda_{m}\right) \quad \Longrightarrow\left(\sup _{\partial B_{d}\left(x_{m}\right)} u_{m} \geq \frac{d^{2}}{2 n}-2 \lambda_{m}\right)
$$


with

$$
\lambda_{m}=C_{0} d^{2-\frac{n}{p}} R^{\frac{n}{p}}\left(\frac{1}{\left|B_{R}\right|} \int_{B_{R}}\left|f_{m}(x)-f_{m}(0)\right|^{p}\right)^{\frac{1}{p}} \leq C_{1} \tau_{m} \quad \text { with } \quad C_{1}=C_{0} d^{2-\frac{n}{p}} R^{\frac{n}{p}}
$$

Passing to the limit as $m \rightarrow+\infty$, we get (up to extraction of a subsequence) that

$$
x_{m} \longrightarrow x_{\infty} \in K
$$

and

$$
\sup _{\partial B_{d}\left(x_{\infty}\right)} u_{\infty} \geq \frac{d^{2}}{2 n}
$$

Contradiction because $u_{\infty}=0$ in a neighbourhood of $K$. This ends the proof of the Lemma.

\subsection{Proof of the decay estimate Proposition 6.2}

\section{Proof of Proposition 6.2}

We perform the proof by contradiction in three Steps. For simplicity, we fix the exponent $p$, and set

$$
\sigma(r)=\sigma_{p}(r / 2)
$$

By the way, we will have to consider sequences of modulus of continuity $\sigma$ that we denote by $\left(\sigma_{m}\right)_{m}$ indexed by $m$, with no possible confusion.

\section{Step 1: A priori estimates on a sequence $v_{m}$}

If the Proposition is false, then there exist sequences $\left(M_{m}\right)_{m},\left(r_{m}\right)_{m},\left(C_{m}\right)_{m},\left(\lambda_{m}\right)_{m},\left(\mu_{m}\right)_{m}$, $\left(f_{m}\right)_{m},\left(u_{m}\right)_{m},\left(\sigma_{m}\right)_{m}$ such that

$$
\left\{\begin{array}{l}
M_{m}, r_{m}, \lambda_{m} \longrightarrow 0 \\
C_{m} \longrightarrow+\infty \\
\mu_{m} \longrightarrow 1
\end{array}\right.
$$

and

$$
M_{m} \geq M\left(u_{m}, r_{m}\right) \geq C_{m} \sigma_{m}\left(r_{m}\right) \quad \text { and } \quad M\left(u_{m}, \lambda_{m} r_{m}\right) \geq \mu_{m} M\left(u_{m}, r_{m}\right)
$$

Let us recall that by assumption $M\left(u_{m}, r_{m}\right)$ is bounded by $M_{m}$ which goes to zero. Therefore there exists $\rho_{m} \in\left(0, \lambda_{m} r_{m}\right]$, such that $N\left(u_{m}, \rho_{m}\right)$ is arbitrarily close to $M\left(u_{m}, \lambda_{m} r_{m}\right)$ and satisfies for instance

$$
\frac{M\left(u_{m}, \lambda_{m} r_{m}\right)}{1+1 / m} \leq N\left(u_{m}, \rho_{m}\right)=: \varepsilon_{m} \leq M\left(u_{m}, r_{m}\right) \leq M_{m} \longrightarrow 0
$$

with

$$
\varepsilon_{m}:=N\left(u_{m}, \rho_{m}\right)=\left(\frac{1}{\rho_{m}^{n+2 p}} \int_{B_{\rho_{m}}}\left|u_{m}-P_{m}\right|^{p}\right)^{\frac{1}{p}} \quad \text { for some } \quad P_{m} \in \mathcal{P}_{\text {reg }}
$$

Now for every $s \in\left(0, s_{m}\right)$ with $s_{m}=r_{m} / \rho_{m} \geq 1 / \lambda_{m} \longrightarrow+\infty$, we have

$$
\inf _{P \in \mathcal{P}}\left(\frac{1}{\left(s \rho_{m}\right)^{n+2 p}} \int_{B_{s \rho_{m}}}\left|u_{m}-P_{m}\right|^{p}\right)^{\frac{1}{p}} \leq \frac{\varepsilon_{m}(1+1 / m)}{\mu_{m}}
$$


Let us set

$$
u_{m}^{\rho_{m}}(x)=u_{m}\left(\rho_{m} \cdot x\right) / \rho_{m}^{2}
$$

We now define the renormalized function

$$
v_{m}(y):=\frac{1}{\varepsilon_{m} \rho_{m}^{2}}\left(u_{m}-P_{m}\right)\left(\rho_{m} y\right)
$$

which satisfies

$$
\left\{\begin{array}{lll}
\Delta v_{m}=g_{m} & \text { on } \quad\left\{u_{m}^{\rho_{m}}>0\right\} \cap\left\{P_{m}>0\right\} \\
v_{m}=0 & \text { on } \quad\left\{u_{m}^{\rho_{m}}=0\right\}^{0} \cap\left\{P_{m}=0\right\}^{0}
\end{array}\right.
$$

where for a set $A$, we denote by $A^{0}$ its interior, and with

$$
g_{m}(y)=\frac{f_{m}\left(\rho_{m} y\right)-f_{m}(0)}{\varepsilon_{m}}
$$

which satisfies for fixed $R \in\left(0, s_{m} / 2\right)$ (as in $(2.22)$ )

$$
\left(\frac{1}{\left|B_{R}\right|} \int_{B_{R}}\left|g_{m}\right|^{p}\right)^{\frac{1}{p}}=\frac{\sigma_{m}\left(2 \rho_{m} R\right)}{\varepsilon_{m}} \leq \frac{1+1 / m}{\mu_{m} C_{m}} \longrightarrow 0 \quad \text { as } \quad m \longrightarrow+\infty
$$

Moreover as in Step 1 of the proof of Propositon 2.5, we get

$$
\inf _{P \in \mathcal{P}}\left(\int_{B_{1}}\left|v_{m}-\left(\frac{P-P_{m}}{\varepsilon_{m}}\right)\right|^{p}\right)^{\frac{1}{p}}=1
$$

and for $s \in\left(0, s_{m}\right)$ :

$$
\inf _{P \in \mathcal{P}}\left(\frac{1}{s^{n+2 p}} \int_{B_{s}}\left|v_{m}-\left(\frac{P-P_{m}}{\varepsilon_{m}}\right)\right|^{p}\right)^{\frac{1}{p}} \leq \frac{1+1 / m}{\mu_{m}} \longrightarrow 1
$$

and for $s \in\left(1, s_{m} / 2\right)$

$$
\left(\frac{1}{s^{n+2 p}} \int_{B_{s}}\left|v_{m}\right|^{p}\right)^{\frac{1}{p}} \leq \frac{C_{1}(1+1 / m)}{\mu_{m}} \ln (2 s) \longrightarrow C_{1} \ln (2 s)
$$

where the limits are taken as $m$ goes to infinity.

We now apply the Caccioppoli type estimate (6.4) to $v_{m}$, and we get for $\operatorname{supp} \zeta \subset B_{R}$ and $W_{m}=v_{m}\left|v_{m}\right|^{\frac{p}{2}-1}$

$$
\int_{\mathbb{R}^{n}} \frac{4(p-1)}{p^{2}} \zeta^{2}\left|\nabla W_{m}\right|^{2} \leq \int_{\mathbb{R}^{n}} \frac{4}{p-1} W_{m}^{2}|\nabla \zeta|^{2}+2\left|B_{R}\right| \frac{\sigma_{m}\left(2 \rho_{m} R\right)}{\varepsilon_{m}}\left(\frac{1}{\left|B_{R}\right|} \int_{B_{R}} \zeta^{2 p^{\prime}}\left|W_{m}\right|^{2}\right)^{\frac{1}{p^{\prime}}}
$$

Step 2: Convergence of the sequence $v_{m}$ 
From (6.44)-(6.45)-(6.46)-(6.47)-(6.48), we get as in Step 2 of the proof of Propositon 2.5 that up to extract a subsequence, we have

$$
\begin{cases}W_{m} \longrightarrow W_{\infty}=v_{\infty}\left|v_{\infty}\right|^{\frac{p}{2}-1} & \text { in } L_{l o c}^{2}\left(\mathbb{R}^{n}\right) \text { and a.e. in } \mathbb{R}^{n} \\ W_{m} \longrightarrow W_{\infty} & \text { weakly in } H_{l o c}^{1}\left(\mathbb{R}^{n}\right)\end{cases}
$$

where $v_{\infty}$ has to be seen as the limit of $v_{m}$.

Up to tilt the coordinates, we can assume that the function $P_{m}$ is fixed with

$$
P_{m}=P_{\infty}=\frac{1}{2}\left(\max \left(0, x_{1}\right)\right)^{2}, \quad \forall m
$$

For any $\beta=\left(\beta_{1}, \ldots, \beta_{n}\right) \in \mathbb{R}^{n}$ and $x=\left(x_{1}, \ldots, x_{n}\right)$, let us define

$$
q_{\beta}(x)=(\beta \cdot x) \cdot \max \left(0, x_{1}\right) .
$$

Then we introduce the following set

$$
T_{P_{\infty}} \mathcal{P}=\left\{q, \quad \exists \beta \in \mathbb{R}^{n}, \quad \text { such that } q=q_{\beta} \quad \text { with } \quad \beta_{1}=0\right\}
$$

which can be interpretated as the tangent space to the set $\mathcal{P}$ at the point $P_{\infty}$, which justifies the notation.

Now for every $q_{\beta} \in T_{P_{\infty}} \mathcal{P}$, we set $\nu_{m}=\frac{e_{1}+\varepsilon_{m} \beta}{\left|e_{1}+\varepsilon_{m} \beta\right|}$ and define

$$
\tilde{P}_{m}:=\frac{1}{2}\left(\max \left(0, \nu_{m} \cdot x\right)\right)^{2}
$$

for which we have

$$
\frac{\tilde{P}_{m}-P_{\infty}}{\varepsilon_{m}} \longrightarrow q_{\beta}
$$

Then (6.45) implies

$$
\inf _{q \in T_{P_{\infty}} \mathcal{P}}\left(\int_{B_{1}}\left|v_{\infty}-q\right|^{p}\right)^{\frac{1}{p}}=1
$$

Moreover (6.46) implies

$$
\inf _{q \in T_{P_{\infty}} \mathcal{P}}\left(\frac{1}{s^{n+2 p}} \int_{B_{s}}\left|v_{\infty}-q\right|^{p}\right)^{\frac{1}{p}} \leq 1 \text { for every } s>0
$$

And (6.47) implies

$$
\left(\frac{1}{s^{n+2 p}} \int_{B_{s}}\left|v_{\infty}\right|^{p}\right)^{\frac{1}{p}} \leq C_{1} \ln (2 s) \quad \text { for every } \quad s \geq 1
$$

and (6.48) implies

$$
\int_{\mathbb{R}^{n}} \frac{4(p-1)}{p^{2}} \zeta^{2}\left|\nabla W_{\infty}\right|^{2} \leq \int_{\mathbb{R}^{n}} \frac{4}{p-1} W_{\infty}^{2}|\nabla \zeta|^{2}
$$


We have $u_{m}^{\rho_{m}}-P_{m}=\varepsilon_{m} v_{m}$, and $\Delta u_{m}^{\rho_{m}}=f_{m}\left(\rho_{m} \cdot\right) \cdot 1_{\left\{u_{m}^{\rho_{m}}>0\right\}}$ where the right hand side is bounded in $L_{l o c}^{p}\left(\mathbb{R}^{n}\right)$. Then by classical elliptic estimates, $u_{m}^{\rho_{m}}$ is bounded in $W_{l o c}^{2, p}\left(\mathbb{R}^{n}\right)$, and then by Sobolev imbeddings, $u_{m}^{\rho_{m}}$ converges (up to extraction of some subsequence) to its limit $P_{\infty}$ in $L_{l o c}^{\infty}\left(\mathbb{R}^{n}\right)$ because $p>n / 2$. We deduce from (6.43) that $v_{\infty}$ satisfies the first line of the following equalities

$$
\left\{\begin{array}{lll}
\Delta v_{\infty}=0 & \text { on } & \left\{P_{\infty}>0\right\}=\left\{y_{1}>0\right\} \\
v_{\infty}=0 & \text { on } & \left\{P_{\infty}=0\right\}^{0}=\left\{y_{1}<0\right\}
\end{array}\right.
$$

To state the second line, we simply apply Lemma 6.5 to the sequence of functions $u_{m}^{\rho_{m}}$ with $\tau_{m}=\sigma_{m}\left(2 \rho_{m} R\right)$ and deduce that for any compact $K$ of $\left\{P_{\infty}=0\right\}^{0} \cap B_{R}$, there exists a constant $C>0$ such that

$$
v_{m} \leq C \frac{\sigma_{m}\left(2 \rho_{m} R\right)}{\varepsilon_{m}} \longrightarrow 0 .
$$

\section{Step 3: Identification of the limit $v_{\infty}$ and contradiction}

From (6.53) and the fact that $v_{\infty}\left|v_{\infty}\right|^{\frac{p}{2}-1}$ belongs to $H_{l o c}^{1}\left(\mathbb{R}^{n}\right)$ we deduce that

$$
v_{\infty}=0 \quad \text { on } \quad\left\{y_{1}=0\right\} .
$$

Because $v_{\infty}$ is harmonic in the half space $\left\{y_{1}>0\right\}$, we deduce from the regularity theory that $v_{\infty}$ is analytic on $\left\{y_{1} \geq 0\right\}$. Therefore we easily check that the function

$$
\tilde{v}_{\infty}(y)=\left\{\begin{array}{cc}
v_{\infty}(y) & \text { if } \quad y_{1} \geq 0 \\
-v_{\infty}\left(-y_{1}, y_{2}, \ldots, y_{n}\right) & \text { if } \quad y_{1}<0
\end{array}\right.
$$

satisfies

$$
\Delta \tilde{v}_{\infty}=0 \quad \text { on } \quad \mathbb{R}^{n}
$$

From (6.51), we see that $\tilde{v}_{\infty} \in \mathcal{S}^{\prime}$ (the dual of the Schwarz space) and then $\tilde{v}_{\infty}$ is a polynomial whose degree is less or equal to 2, still from (6.51). Moreover from (6.50) we deduce that $\tilde{v}_{\infty}$ is homogeneous of degree 2 .

From (6.54), we deduce with $y^{\prime}=\left(y_{2}, \ldots, y_{n}\right)$ that

$$
\tilde{v}_{\infty}\left(y_{1}, y^{\prime}\right)=\tilde{v}_{\infty}\left(0, y^{\prime}\right)+y_{1} \frac{\partial \tilde{v}_{\infty}}{\partial y_{1}}\left(0, y^{\prime}\right)+\frac{1}{2} y_{1}^{2} \frac{\partial \tilde{v}_{\infty}}{\partial y_{1}^{2}}\left(0, y^{\prime}\right)
$$

with $\tilde{v}_{\infty}\left(0, y^{\prime}\right)=0, \frac{\partial \tilde{v}_{\infty}}{\partial y_{1}}\left(0, y^{\prime}\right)=\beta \cdot y$ for some $\beta \in \mathbb{R}^{n}$ with $\beta_{1}=0, \frac{\partial \tilde{v}_{\infty}}{\partial y_{1}^{2}}\left(0, y^{\prime}\right)=$ constant $=\Delta \tilde{v}_{\infty}=0$, i.e.

$$
\tilde{v}_{\infty}\left(y_{1}, y^{\prime}\right)=y_{1} \cdot(\beta \cdot y)
$$

and

$$
v_{\infty}=p_{\beta}
$$

This gives a contradiction with (6.49).

This ends the proof of the Proposition. 


\section{Singular points of the free boundary and proof of Theorem 1.11}

\subsection{Monotonicity formula for singular points and proof of Theo- rem 1.10}

\section{Proposition 7.1 (Monotonicity formula for singular points)}

Let $p \in(\max (n / 2,1),+\infty)$ with $p \geq 2 n /(n+1)$. There exists a constant $C>0$. For any matrix $Q \in \mathbb{R}_{\text {sym }}^{n \times n}$ with trace $Q=1$ and $Q \geq 0$, we set $v(x)=\frac{1}{2} t x \cdot Q \cdot x$. Then, for any solution $u$ of (1.8), we have

$$
\frac{d}{d r}\left(\frac{1}{r^{n+3}} \int_{\partial B_{r}}(u-v)^{2}\right)=g(r)+\frac{4}{r} \int_{B_{r}} \frac{1}{|x|^{n}}\left|\frac{U(x)}{|x|^{2}}\right|^{2}
$$

for $U=x \cdot \nabla u-2 u$ and

$$
-g(r) \leq C \frac{\Sigma_{p}(r)}{r} F_{p^{\prime}}(r) \quad \text { with } \quad \Sigma_{p}(r)=\sigma_{p}(r)+\int_{0}^{r} \frac{\sigma_{p}(s)}{s} d s
$$

and for $\frac{1}{p}+\frac{1}{p^{\prime}}=1$

$$
F_{p^{\prime}}(r)=\left(\frac{1}{\left|B_{r}\right|} \int_{B_{r}}\left|\frac{u-v}{r^{2}}\right|^{p^{\prime}}\right)^{\frac{1}{p^{\prime}}}+\sup _{\rho \in(0, r]}\left(\frac{1}{\left|B_{\rho}\right|} \int_{B_{\rho}}\left|\frac{U}{\rho^{2}}\right|^{p^{\prime}}\right)^{\frac{1}{p^{\prime}}}
$$

Moreover there exists a constant $C_{0}>0$ such that

$$
\forall r \in(0,1 / 2), \quad F_{p^{\prime}}(r) \leq C_{0}\left\{\left(\int_{B_{1}}|u|^{p}\right)^{\frac{1}{p}}+\sigma_{p}(1)+1\right\}
$$

\section{Proof of Proposition 7.1}

From the appendix of [32], we have

$$
\frac{d}{d r}\left(\frac{1}{r^{n+3}} \int_{\partial B_{r}}(u-v)^{2}\right)=\frac{2}{r}\left(\Phi(r)-\Phi\left(0^{+}\right)\right)+\frac{2}{r^{n+3}} \int_{B_{r} \cap\{u>0\}}(u-v)(f-f(0))+\frac{2}{r^{n+3}} \int_{B_{r} \cap\{u=0\}} v
$$

From (4.39), we deduce (7.55) with

$$
g(r)=-\frac{4}{r} \int_{0}^{r} \frac{d s}{s^{n+3}} \int_{B_{s}} U(f(x)-f(0))+\frac{2}{r^{n+3}} \int_{B_{r} \cap\{u>0\}}(u-v)(f-f(0))+\frac{2}{r^{n+3}} \int_{B_{r} \cap\{u=0\}} v
$$

for $U=x \cdot \nabla u-2 u$. The result follows from Proposition 1.5 and Corollary 4.1 (noticing moreover that the last term in $g$ is non-negative because $v \geq 0$ ). This ends the proof of the Proposition.

\section{Corollary 7.2 (Uniqueness of the blow-up limits)}

Under the assumptions of Proposition 7.1, let us consider

$$
u^{\varepsilon}(x)=\frac{u(\varepsilon x)}{\varepsilon^{2}}
$$

If we assume that $\sigma_{p}$ is double Dini, then $u^{\varepsilon}$ converges (uniformly on compact sets) to a unique limit $u^{0}=v$ as $\varepsilon$ goes to zero, for some $v$ as in Proposition 7.1. 


\section{Proof of Corollary 7.2}

Let us set

$$
\Psi_{u}^{v}(r)=\frac{1}{r^{n+3}} \int_{\partial B_{r}}(u-v)^{2}
$$

Let us call $u^{0}$ one of the blow-up limits of $u^{\varepsilon}$. Then, using the 2-homogeneity of $u^{0}$, we get

$$
\Psi_{u}^{u^{0}}(\varepsilon r)=\Psi_{u^{\varepsilon}}^{u^{0}}(r) \longrightarrow \Psi_{u^{0}}^{u^{0}}(r)=0
$$

where the convergence happens for a suitable subsequence. On the other hand, from Proposition 7.1, we deduce that for double Dini $\sigma_{p}$, the following limit exists

$$
\lim _{\rho \rightarrow 0} \Psi_{u}^{u^{0}}(\rho)=\Psi_{u}^{u^{0}}\left(0^{+}\right)
$$

We conclude that

$$
\Psi_{u}^{u^{0}}\left(0^{+}\right)=0
$$

and then the convergence in (7.57) happens for the whole sequence as $\varepsilon$ goes to zero. The convergence on compact sets of $\mathbb{R}^{n}$ follows. This ends the proof of the Corollary.

When we assume moreover that $p \geq 2$, we get a better estimate than in Proposition 7.1, namely

Proposition 7.3 (Monotonicity formula for singular points for $p \geq 2$ )

Let $p \in(\max (n / 2,1),+\infty)$ with $p \geq 2 n /(n+1)$ and $p \geq 2$. There exists a constant $C>0$. For any matrix $Q \in \mathbb{R}_{\text {sym }}^{n \times n}$ with trace $Q=1$ and $Q \geq 0$, we set $v(x)=\frac{1}{2}^{t} x \cdot Q \cdot x$. Then, for any solution $u$ of (1.8), we have

$$
\frac{d}{d r}\left(\frac{1}{r^{n+3}} \int_{\partial B_{r}}(u-v)^{2}\right)=h(r)+\frac{2}{r} \int_{B_{r}} \frac{1}{|x|^{n}}\left|\frac{U(x)}{|x|^{2}}\right|^{2}
$$

for $U=x \cdot \nabla u-2 u$ and

$$
-h(r) \leq C\left\{\frac{1}{r} \int_{0}^{r} \frac{\sigma_{p}^{2}(s)}{s} d s+\frac{\sigma_{p}(r)}{r}\left(\frac{1}{\left|B_{r}\right|} \int_{B_{r}}\left|\frac{u-v}{r^{2}}\right|^{p^{\prime}}\right)^{\frac{1}{p^{\prime}}}\right\}
$$

and for $\frac{1}{p}+\frac{1}{p^{\prime}}=1$. Moreover there exists a constant $C_{0}>0$ such that

$$
\forall r \in(0,1 / 2), \quad\left(\frac{1}{\left|B_{r}\right|} \int_{B_{r}}\left|\frac{u-v}{r^{2}}\right|^{p^{\prime}}\right)^{\frac{1}{p^{\prime}}} \leq C_{0}\left\{\left(\int_{B_{1}}|u|^{p}\right)^{\frac{1}{p}}+\sigma_{p}(1)+1\right\}
$$

\section{Proof of Proposition 7.3}

We start with

$$
h(r)=g(r)+\frac{2}{r} \int_{B_{r}} \frac{1}{|x|^{n}}\left|\frac{U(x)}{|x|^{2}}\right|^{2}
$$


where $g$ is given in (7.56). We really have to work on the following term (first term in $g$ given in (7.56), using the fact that $p^{\prime} \leq 2$ :

$$
\begin{aligned}
\frac{4}{r} \int_{0}^{r} \frac{d s}{s^{n+3}} \int_{B_{s}} U(f(x)-f(0)) & \leq \frac{4\left|B_{1}\right|}{r} \int_{0}^{r} \frac{d s}{s} \sigma_{p}(s)\left(\frac{1}{\left|B_{s}\right|} \int_{B_{s}}\left|\frac{U}{s^{2}}\right|^{p^{\prime}}\right)^{\frac{1}{p^{\prime}}} \\
& \leq \frac{4\left|B_{1}\right|}{r} \int_{0}^{r} \frac{d s}{s} \sigma_{p}(s)\left(\frac{1}{\left|B_{s}\right|} \int_{B_{s}}\left|\frac{U}{s^{2}}\right|^{2}\right)^{\frac{1}{2}} \\
& \leq \frac{2\left|B_{1}\right|}{r}\left\{\frac{1}{\varepsilon} \int_{0}^{r} \frac{d s}{s} \sigma_{p}^{2}(s)+\varepsilon \int_{0}^{r} \frac{d s}{s}\left(\frac{1}{\left|B_{s}\right|} \int_{B_{s}}\left|\frac{U}{s^{2}}\right|^{2}\right)\right\}
\end{aligned}
$$

for any $\varepsilon>0$ that we will choose later small enough.

We now claim that

$$
\int_{0}^{r} \frac{d s}{s}\left(\frac{1}{\left|B_{s}\right|} \int_{B_{s}}\left|\frac{U}{s^{2}}\right|^{2}\right) \leq \frac{1}{(n+4)\left|B_{1}\right|} \int_{B_{r}} \frac{1}{|x|^{n}}\left|\frac{U(x)}{|x|^{2}}\right|^{2}
$$

Choosing now $\varepsilon$ small enough, we see that the term $\frac{2\left|B_{1}\right| \varepsilon}{r} \int_{0}^{r} \frac{d s}{s}\left(\frac{1}{\left|B_{s}\right|} \int_{B_{s}}\left|\frac{U}{s^{2}}\right|^{2}\right)$ is controled by the term $\frac{2}{r} \int_{B_{r}} \frac{1}{|x|^{n}}\left|\frac{U(x)}{|x|^{2}}\right|^{2}$ introduced in the definition (7.59) of $h$. The expected bound on $-h$ then follows easily.

We only need to prove (7.60). We compute (with obvious notation)

$$
\begin{aligned}
\int_{0}^{r} \frac{d s}{s}\left(\frac{1}{\left|B_{s}\right|} \int_{B_{s}}\left|\frac{U}{s^{2}}\right|^{2}\right) & =\frac{1}{\left|B_{1}\right|} \int_{\mathbf{S}^{n-1}} d \theta \int_{0}^{r} \frac{d s}{s^{n+5}} \int_{0}^{s} d \rho \rho^{n-1}|U(\rho, \theta)|^{2} \\
& =\frac{1}{\left|B_{1}\right|} \int_{\mathbf{S}^{n-1}} d \theta \int_{0}^{r} d \rho \rho^{n-1}|U(\rho, \theta)|^{2} \int_{\rho}^{r} \frac{d s}{s^{n+5}} \\
& \leq \frac{1}{(n+4)\left|B_{1}\right|} \int_{\mathbf{S}^{n-1}} d \theta \int_{0}^{r} \frac{d \rho}{\rho^{5}}|U(\rho, \theta)|^{2} \\
& =\frac{1}{(n+4)\left|B_{1}\right|} \int_{B_{r}} \frac{1}{|x|^{n}}\left|\frac{U(x)}{|x|^{2}}\right|^{2}
\end{aligned}
$$

This ends the proof of the Proposition.

As a corollary, we see that Corollary 7.2 holds if we assume moreover $p \geq 2$, but relax the condition on $\sigma_{p}$ to be only Dini. This is the following proof.

\section{Proof of Theorem 1.10}

This is a consequence of Proposition 7.3, noting in particular that

$$
\int_{0}^{1} \frac{d r}{r}\left(\int_{0}^{r} \frac{\sigma_{p}^{2}(s)}{s} d s\right) \leq\left(\int_{0}^{1} \frac{\sigma_{p}^{2}(s)}{s} d s\right)^{2}<+\infty
$$


This ends the proof of the Theorem.

\subsection{Proof of Theorem 1.11}

Here we adapt the proof of Theorem 1.8, and replace the set $\mathcal{P}_{\text {reg }}$ by the set

$$
\mathcal{P}_{\text {sing }}=\left\{P, \quad \exists Q \in \mathbb{R}_{\text {sym }}^{n \times n}, \quad P=\frac{1}{2}{ }^{t} x \cdot Q \cdot x, \quad \operatorname{trace}(Q)=1, \quad Q \geq 0\right\}
$$

keeping the same notation for $M(u, \rho)$ and $N(u, r)$ (see Definition 2.2). Here the set $\mathcal{P}_{\text {sing }}$ contains the possible limit behaviours of the solution at the origin when the origin is a singular point. Again $M$ is finite for $p \in(\max (n / 2,1),+\infty)$ by Proposition 1.5.

Then we have the following cornerstone result which will be proved in subsection 7.4.

\section{Proposition 7.4 (Decay estimate in a smaller ball)}

Given $p \in(\max (n / 2,1),+\infty)$ with $p \geq 2 n /(n+1)$ and $p \geq 2$, there exist constants $M_{0}, C_{0}>$ $0, r_{0}, \lambda, \mu \in(0,1)$ (only depending on $p$ and the dimension $n$ ) such that for every functions $u$ and $f$ satisfying (1.8) with a modulus of continuity $\sigma_{p}$ given by (1.9), then we have the following property

$$
\forall r \in\left(0, r_{0}\right), \quad\left(M(u, r) \leq M_{0}\right) \Longrightarrow\left(M(u, \lambda r)<\mu M(u, r) \quad \text { or } \quad M(u, r)<C_{0} \tilde{\Sigma}_{p}(r / 2)\right)
$$

where

$$
\tilde{\Sigma}_{p}(r)=\sigma_{p}(r)+\left(\int_{0}^{r} d s \frac{\sigma_{p}^{2}(s)}{s}\right)^{\frac{1}{2}}
$$

Remark 7.5 Because the problem is nonlinear here, so $M(u, r)$ has to be small enough (less than the threshold $M_{0}$ ) to satisfy the decay estimate. Just think to the case of blowup limits $u^{0}$ at regular points, like for instance $u^{0}(x)=\frac{1}{2}\left(\max \left(0, x_{1}\right)\right)^{2}$ for which we have $M\left(u^{0}, r\right)>M_{0}$.

\section{Proof of Theorem 1.11}

The proof of Theorem 1.11 follows exactly the lines of the proof of Theorem 1.8. This ends the proof of the Theorem.

\subsection{Preliminary results}

To prove Proposition 7.4, we will need the following results

\section{Lemma 7.6 (Liouville result (I))}

Let $v_{\infty} \in H_{\text {loc }}^{1}\left(\mathbb{R}^{n}\right)$ satisfying $\Delta v_{\infty} \leq 0$ on $\mathbb{R}^{n}$ and harmonic on $\mathbb{R}^{n} \backslash\left\{x_{1}=0\right\}$. If $v_{\infty}$ is homogeneous of degree 2 , then $\Delta v_{\infty}=0$.

\section{Proof of Lemma 7.6}

By assumption, we know that $\Delta v_{\infty}$ is a non-positive measure $\mu$ supported in $\left\{x_{1}=0\right\}$ (which 
is moreover invariant by dilations). Now let $P$ be a $C^{2}$ and 2-homogeneous function, and $\psi \in C_{c}^{\infty}([0,+\infty)$ with $\psi \geq 0$ and $\Psi(x)=\psi(|x|)$. Then we compute

$$
\begin{aligned}
-<\mu, \Psi P> & =<-\Delta v_{\infty}, \Psi P> \\
& =\int_{\mathbb{R}^{n}} \nabla v_{\infty} \cdot \nabla(\Psi P) \\
& =\int_{\mathbb{R}^{n}} \Psi \nabla v_{\infty} \cdot \nabla P+P \nabla v_{\infty} \cdot \nabla \Psi \\
& =\int_{\mathbb{R}^{n}}-\Psi v_{\infty} \Delta P-v_{\infty} \nabla \Psi \cdot \nabla P+P \nabla v_{\infty} \cdot \nabla \Psi \\
& =\int_{\mathbb{R}^{n}}-\Psi v_{\infty} \Delta P
\end{aligned}
$$

where in the last line we have used the homogeneity which implies that $x \cdot \nabla P(x)=2 P(x)$ and the same property for $v_{\infty}$. Let us choose $P(x)=-(n-1) x_{1}^{2}+x_{2}^{2}+\ldots+x_{n}^{2}$. Then $\Delta P=0$ and we get

$$
-<\mu, \Psi P>=0
$$

This implies that supp $\mu \subset\{0\}$, and then

$$
\mu=-c \delta_{0} \quad \text { for some } \quad c \geq 0
$$

Finally the invariance of $\mu$ by dilations implies that $c=0$ and $\mu=0$, i.e. $\Delta v_{\infty}=0$. This ends the proof of the Lemma.

\section{Lemma 7.7 (Liouville result (II))}

Let $v_{\infty}$ be a harmonic polynomial on $\mathbb{R}^{n}$, homogeneous of degree 2 , and satisfying the following conditions for some $\bar{k}_{\infty} \in\{1, \ldots, n\}$

$$
\left\{\begin{array}{l}
v_{\infty} \geq 0 \text { on } \quad\left\{x_{i}=0, \quad i=1, \ldots, \bar{k}_{\infty}\right\} \\
\int_{B_{1}} v_{\infty}\left(\sum_{i=1}^{n} \gamma_{i} x_{i}^{2}\right) \leq 0 \quad \text { for every }\left\{\begin{array}{l}
\gamma=\left(\gamma_{1}, \ldots, \gamma_{n}\right) \in \mathbb{R}^{\bar{k}_{\infty}} \times[0,+\infty)^{n-\bar{k}_{\infty}} \\
\text { with } \sum_{i=1}^{n} \gamma_{i}=0
\end{array}\right. \\
\int_{B_{1}} v_{\infty} x_{i} x_{j}=0 \quad \text { for } i=1, \ldots, \bar{k}_{\infty}, \quad j=1, \ldots, n, \quad j \neq i
\end{array}\right.
$$

Then $v_{\infty}=0$.

We first prove the following result

\section{Lemma 7.8 (Scalar product)}

Let $V_{n}$ the space of symmetric $n \times n$ matrices $Q$ such that trace $Q=0$. Then there exists $\beta>0$ such that

$$
\forall Q \in V_{n}, \quad \frac{1}{\left|B_{1}\right|} \int_{B_{1}}\left({ }^{t} x \cdot Q \cdot x\right)^{2}=\beta \cdot \operatorname{trace}\left({ }^{t} Q \cdot Q\right)
$$




\section{Proof of Lemma 7.8}

Given a matrix $Q \in V_{n}$, we set

$$
(Q, Q)=\frac{1}{\left|B_{1}\right|} \int_{B_{1}}\left(\frac{1}{2} t x \cdot Q \cdot x\right)^{2}
$$

We then diagonalize the matrix $Q$ in an orthonormal basis with coordinates $\left(x_{1}, \ldots, x_{n}\right)$, and with eigenvalues $\left(\lambda_{1}, \ldots, \lambda_{n}\right)$. Therefore we can compute

$$
(Q, Q)=\frac{1}{\left|B_{1}\right|} \int_{B_{1}}\left(\sum_{i=1}^{n} \lambda_{i} x_{i}^{2}\right)^{2}=\sum_{i, j=1}^{n} a_{i j} \lambda_{i} \lambda_{j}
$$

where

$$
a_{i j}=b+(a-b) \delta_{i j} \quad \text { with } \quad b=\frac{1}{\left|B_{1}\right|} \int_{B_{1}} x_{1}^{2} x_{2}^{2}, \quad a=\frac{1}{\left|B_{1}\right|} \int_{B_{1}} x_{1}^{4}
$$

We deduce that

$$
(Q, Q)=b\left(\sum_{i=1}^{n} \lambda_{i}\right)^{2}+(a-b) \sum_{i=1}^{n} \lambda_{i}^{2}
$$

and then using the fact that $\sum_{i=1}^{n} \lambda_{i}=0$, and $\beta=a-b$

$$
(Q, Q)=\beta \operatorname{trace}\left({ }^{t} Q \cdot Q\right)
$$

Finally $\beta>0$ because $(Q, Q)$ is trivially non-zero for non-zero $Q$. This ends the proof of the Lemma.

\section{Proof of Lemma 7.7}

We first remark that we can write $v_{\infty}(x)=\frac{1}{2}^{t} x \cdot C \cdot x$ with $C \in V_{n}$ (using the notation $V_{n}$ as in Lemma 7.8 to denote the symmetric $n \times n$ matrices with trace equal to 0). From (7.62), and the fact that the matrices $\frac{1}{2}\left(e_{i} \otimes e_{j}+e_{j} \otimes e_{i}\right)$ for $i \neq j$ are orthogonal for the scalar product studied in Lemma 7.8 , we see that we can write

$$
C=\left(\begin{array}{ll}
A & 0 \\
0 & B
\end{array}\right)
$$

with $A$ a diagonal matrix $\bar{k}_{\infty} \times \bar{k}_{\infty}$ and $B$ a non-negative matrix $\left(n-\bar{k}_{\infty}\right) \times\left(n-\bar{k}_{\infty}\right)$. Let choose $\gamma=\left(\gamma^{\prime}, \gamma^{\prime \prime}\right) \in(-\infty, 0)^{\bar{k}_{\infty}} \times(0,+\infty)^{n-\bar{k}_{\infty}}$ in (7.62) with identical constant coordinates $\gamma^{\prime}=\left(-\gamma_{A}, \ldots,-\gamma_{A}\right)$ and $\gamma^{\prime \prime}=\left(\gamma_{B}, \ldots, \gamma_{B}\right)$, satisfying $\gamma_{A}>0, \gamma_{B}>0$, and $-\bar{k}_{\infty} \gamma_{A}+(n-$ $\left.\bar{k}_{\infty}\right) \gamma_{B}=0$. Then we get from $(7.62)$

$$
-\gamma_{A}(\operatorname{trace} A)+\gamma_{B}(\operatorname{trace} B) \leq 0
$$

Because trace $C=0$ and trace $B \geq 0$, we deduce that trace $A \leq 0$, and then trace $A=$ $0=$ trace $B$. Therefore $B=0$. Choosing now any $\gamma=\left(\gamma^{\prime}, \gamma^{\prime \prime}\right)$ in $(7.62)$ with $\gamma^{\prime \prime}=0$ and $\sum_{i=1}^{\bar{k}_{\infty}} \gamma_{i}^{\prime}=0$, we deduce that

$$
\forall \bar{A} \in V_{\bar{k}_{\infty}}, \quad \operatorname{trace}^{t} A \bar{A}=0
$$

which implies that $A=0$, because $A$ itself satisfies trace $A=0$ belongs to $V_{\bar{k}_{\infty}}$. This ends the proof of the Lemma. 


\subsection{Proof of the decay estimate Proposition 7.4}

\section{Proof of Proposition 7.4}

We perform the proof by contradiction in five Steps. For simplicity, we set

$$
\sigma(r)=\sigma_{p}(r / 2)
$$

and

$$
\tilde{\Sigma}(r)=\sigma(r)+\left(\int_{0}^{r} d s \frac{\sigma^{2}(s)}{s}\right)^{\frac{1}{2}}
$$

By the way, we will have to consider sequences of modulus of continuity $\sigma$ that we denote by $\left(\sigma_{m}\right)_{m}$ indexed by $m$, with no possible confusion.

Similarly, we will associate their $\left(\tilde{\Sigma}_{m}\right)_{m}$, using formula (7.63).

Step 1: A priori estimates on a sequence $v_{m}$

If the Proposition is false, then there exist sequences $\left(M_{m}\right)_{m},\left(r_{m}\right)_{m},\left(C_{m}\right)_{m},\left(\lambda_{m}\right)_{m},\left(\mu_{m}\right)_{m}$, $\left(f_{m}\right)_{m},\left(u_{m}\right)_{m},\left(\sigma_{m}\right)_{m}$ such that

$$
\left\{\begin{array}{l}
M_{m}, r_{m}, \lambda_{m} \longrightarrow 0 \\
C_{m} \longrightarrow+\infty \\
\mu_{m} \longrightarrow 1
\end{array}\right.
$$

and

$$
M_{m} \geq M\left(u_{m}, r_{m}\right) \geq C_{m} \tilde{\Sigma}_{m}\left(r_{m}\right) \quad \text { and } \quad M\left(u_{m}, \lambda_{m} r_{m}\right) \geq \mu_{m} M\left(u_{m}, r_{m}\right)
$$

Let us recall that by assumption $M\left(u_{m}, r_{m}\right)$ is bounded by $M_{m}$ which goes to zero. Therefore there exists $\rho_{m} \in\left(0, \lambda_{m} r_{m}\right]$, such that $N\left(u_{m}, \rho_{m}\right)$ is arbitrarily close to $M\left(u_{m}, \lambda_{m} r_{m}\right)$ and satisfies for instance

$$
\frac{M\left(u_{m}, \lambda_{m} r_{m}\right)}{1+1 / m} \leq N\left(u_{m}, \rho_{m}\right)=: \varepsilon_{m} \leq M\left(u_{m}, r_{m}\right) \leq M_{m} \longrightarrow 0
$$

with

$$
\varepsilon_{m}:=N\left(u_{m}, \rho_{m}\right)=\left(\frac{1}{\rho_{m}^{n+4}} \int_{B_{\rho_{m}}}\left|u_{m}-P_{m}\right|^{2}\right)^{\frac{1}{2}} \quad \text { for some } \quad P_{m} \in \mathcal{P}_{\text {sing }}
$$

Now for every $s \in\left(0, s_{m}\right)$ with $s_{m}=r_{m} / \rho_{m} \geq 1 / \lambda_{m} \longrightarrow+\infty$, we have

$$
\inf _{P \in \mathcal{P}_{\text {sing }}}\left(\frac{1}{\left(s \rho_{m}\right)^{n+4}} \int_{B_{s \rho_{m}}}\left|u_{m}-P_{m}\right|^{2}\right)^{\frac{1}{2}} \leq \frac{\varepsilon_{m}(1+1 / m)}{\mu_{m}}
$$

Let us set

$$
u_{m}^{\rho_{m}}(x)=u_{m}\left(\rho_{m} \cdot x\right) / \rho_{m}^{2}
$$

We now define the renormalized function

$$
v_{m}(y):=\frac{1}{\varepsilon_{m} \rho_{m}^{2}}\left(u_{m}-P_{m}\right)\left(\rho_{m} y\right)
$$


which satisfies

$$
\Delta v_{m}=g_{m} \quad \text { on } \quad\left\{u_{m}^{\rho_{m}}>0\right\}
$$

and

$$
\Delta v_{m} \leq g_{m} \cdot 1_{\left\{u_{m}^{\rho m}>0\right\}} \quad \text { on } \quad \mathbb{R}^{n}
$$

with

$$
g_{m}(y)=\frac{f_{m}\left(\rho_{m} y\right)-f_{m}(0)}{\varepsilon_{m}}
$$

which satisfies for fixed $R \in\left(0, s_{m} / 2\right)$ (as in $(2.22)$ )

$$
\left(\frac{1}{\left|B_{R}\right|} \int_{B_{R}}\left|g_{m}\right|^{p}\right)^{\frac{1}{p}}=\frac{\sigma_{m}\left(2 \rho_{m} R\right)}{\varepsilon_{m}} \leq \frac{1+1 / m}{\mu_{m} C_{m}} \longrightarrow 0 \quad \text { as } \quad m \longrightarrow+\infty
$$

Moreover as in Step 1 of the proof of Propositon 2.5, we get

$$
\inf _{P \in \mathcal{P}_{\text {sing }}}\left(\int_{B_{1}}\left|v_{m}-\left(\frac{P-P_{m}}{\varepsilon_{m}}\right)\right|^{2}\right)^{\frac{1}{2}}=1,
$$

and for $s \in\left(0, s_{m}\right)$ :

$$
\inf _{P \in \mathcal{P}_{\text {sing }}}\left(\frac{1}{s^{n+2 p}} \int_{B_{s}}\left|v_{m}-\left(\frac{P-P_{m}}{\varepsilon_{m}}\right)\right|^{2}\right)^{\frac{1}{2}} \leq \frac{1+1 / m}{\mu_{m}} \longrightarrow 1
$$

and for $s \in\left(1, s_{m} / 2\right)$

$$
\left(\frac{1}{s^{n+2 p}} \int_{B_{s}}\left|v_{m}\right|^{2}\right)^{\frac{1}{2}} \leq \frac{C_{1}(1+1 / m)}{\mu_{m}} \ln (2 s) \longrightarrow C_{1} \ln (2 s)
$$

where the limits are taken as $m$ goes to infinity.

We now apply the Caccioppoli type estimate (6.4) to $v_{m}$ with $p=2$, and get for $\operatorname{supp} \zeta \subset$ $B_{R}$

$$
\int_{\mathbb{R}^{n}} \zeta^{2}\left|\nabla v_{m}\right|^{2} \leq \int_{\mathbb{R}^{n}} 4 v_{m}^{2}|\nabla \zeta|^{2}+2\left|B_{R}\right| \frac{\sigma_{m}\left(2 \rho_{m} R\right)}{\varepsilon_{m}}\left(\frac{1}{\left|B_{R}\right|} \int_{B_{R}} \zeta^{4}\left|v_{m}\right|^{2}\right)^{\frac{1}{2}}
$$

\section{Step 2: Convergence of the sequence $v_{m}$}

From (7.67)-(7.68)-(7.69)-(7.70)-(7.71), we get as in Step 2 of the proof of Propositon 2.5 that up to extract a subsequence, we have

$$
\begin{cases}v_{m} \longrightarrow v_{\infty} & \text { in } L_{l o c}^{2}\left(\mathbb{R}^{n}\right) \text { and a.e. in } \mathbb{R}^{n} \\ v_{m} \longrightarrow v_{\infty} & \text { weakly in } H_{l o c}^{1}\left(\mathbb{R}^{n}\right)\end{cases}
$$

Then (7.68) implies

$$
\left(\int_{B_{1}}\left|v_{\infty}\right|^{2}\right)^{\frac{1}{2}}=1
$$


and (7.70) implies

$$
\left(\frac{1}{s^{n+4}} \int_{B_{s}}\left|v_{\infty}\right|^{2}\right)^{\frac{1}{2}} \leq C_{1} \ln (2 s) \quad \text { for every } \quad s \geq 1
$$

and (7.71) implies

$$
\int_{\mathbb{R}^{n}} \zeta^{2}\left|\nabla v_{\infty}\right|^{2} \leq \int_{\mathbb{R}^{n}} 4 v_{\infty}^{2}|\nabla \zeta|^{2}
$$

Up to extract a subsequence we can assume that $P_{m}$ converges to some $P_{\infty}$.

We have $u_{m}^{\rho_{m}}-P_{m}=\varepsilon_{m} v_{m}$, and $\Delta u_{m}^{\rho_{m}}=f_{m}\left(\rho_{m} \cdot\right) \cdot 1_{\left\{u_{m}^{\rho_{m}}>0\right\}}$ where the right hand side is bounded in $L_{l o c}^{p}\left(\mathbb{R}^{n}\right)$, then by classical elliptic estimates, $u_{m}^{\rho_{m}}$ is bounded in $W_{l o c}^{2, p}\left(\mathbb{R}^{n}\right)$, and then by Sobolev imbeddings, $u_{m}^{\rho_{m}}$ converges (up to extraction of some subsequence) to its limit $P_{\infty}$ in $L_{l o c}^{\infty}\left(\mathbb{R}^{n}\right)$ because $p>n / 2$. Then we deduce from (7.65) that $v_{\infty}$ satisfies

$$
\Delta v_{\infty}=0 \quad \text { on } \quad\left\{P_{\infty}>0\right\}
$$

Moreover we also deduce from (7.66) that

$$
\Delta v_{\infty} \leq 0 \quad \text { on } \quad \mathbb{R}^{n}
$$

\section{Step 3: The limit differential}

We define the following limit differential (up to a subsequence):

$\bar{\partial}_{P_{\infty}} \mathcal{P}_{\text {sing }}=\left\{q=\frac{1}{2}{ }^{t} x \cdot Q \cdot x, \quad \exists Q \in \mathbb{R}_{\text {sym }}^{n \times n}, \quad \operatorname{trace}(Q)=0, \quad \exists \bar{P}_{m} \in \mathcal{P}_{\text {sing }} \quad\right.$ with $\left.\quad \frac{\bar{P}_{m}-P_{m}}{\varepsilon_{m}} \longrightarrow q\right\}$

By construction $\bar{\partial}_{P_{\infty}} \mathcal{P}_{\text {sing }}$ contains the origin and is convex, because $\mathcal{P}_{\text {sing }}$ is convex. Moreover it is easy to check that $\bar{\partial}_{P_{\infty}} \mathcal{P}_{\text {sing }}$ is closed.

Then (7.68) implies

$$
\inf _{q \in \bar{\partial}_{P_{\infty}} \mathcal{P}_{\text {sing }}}\left(\int_{B_{1}}\left|v_{\infty}-q\right|^{p}\right)^{\frac{1}{p}}=1
$$

and (7.69) implies

$$
\inf _{q \in \bar{\partial}_{P_{\infty}} \mathcal{P}_{\text {sing }}}\left(\frac{1}{s^{n+2 p}} \int_{B_{s}}\left|v_{\infty}-q\right|^{p}\right)^{\frac{1}{p}} \leq 1 \quad \text { for every } \quad s>0
$$

\section{Step 4: 2-homogeneity of $v_{\infty}$ and consequences}

To deduce the 2-homogeneity of $v_{\infty}$, we will make strong use of the monotonicity formula (Proposition 7.3) for singular points in the case $p \geq 2$.

Comming back at the level of the functions $v_{m}$, we remark that Proposition 7.3 implies

$$
\frac{d}{d r}\left(\frac{1}{r^{n+3}} \int_{\partial B_{r}} v_{m}^{2}\right)=h_{m}(r)+\frac{2}{r} \int_{B_{r}} \frac{1}{|x|^{n}}\left|\frac{U_{m}(x)}{|x|^{2}}\right|^{2}
$$


for $U_{m}=x \cdot \nabla v_{m}-2 v_{m}$ and (because $p^{\prime} \leq 2 \leq p$ )

$$
-h_{m}(r) \leq C\left\{\frac{1}{r} \int_{0}^{r} \frac{\sigma_{m}^{2}\left(2 \rho_{m} s\right)}{\varepsilon_{m}^{2} s} d s+\frac{\sigma_{m}\left(2 \rho_{m} r\right)}{\varepsilon_{m} r}\left(\frac{1}{\left|B_{r}\right|} \int_{B_{r}}\left|\frac{v_{m}}{r^{2}}\right|^{p}\right)^{\frac{1}{p}}\right\}
$$

In particular, we have with $s_{m} \longrightarrow+\infty$

$$
\frac{\tilde{\Sigma}_{m}}{\varepsilon_{m}}=\frac{\sigma_{m}\left(\rho_{m} s_{m}\right)}{\varepsilon_{m}}+\left(\int_{0}^{\frac{s_{m}}{2}} \frac{\sigma_{m}^{2}\left(2 \rho_{m} s\right)}{\varepsilon_{m}^{2} s} d s\right)^{\frac{1}{2}} \leq \frac{(1+1 / m)}{\mu_{m} C_{m}} \longrightarrow 0
$$

Therefore using the notation $a^{+}=\max (a, 0)$, we get as $m \longrightarrow+\infty$

$$
\left(-h_{m}\right)^{+} \longrightarrow 0 \text { uniformly on compact sets of }(0,+\infty)
$$

Let now $\varphi \in C_{c}^{\infty}(0,+\infty)$ such that $\varphi \geq 0$. Multiplying (7.79) by $\varphi$ and integrating by parts, we get

$$
-\int_{0}^{+\infty} d r \varphi^{\prime}(r)\left(\frac{1}{r^{n+3}} \int_{\partial B_{r}} v_{m}^{2}\right) \geq \int_{0}^{+\infty} d r \varphi(r)\left\{\frac{2}{r} \int_{B_{r}} \frac{1}{|x|^{n}}\left|\frac{U_{m}(x)}{|x|^{2}}\right|^{2}-\left(-h_{m}(r)\right)^{+}\right\}
$$

Passing to the limit in (7.80), we get

$$
-\int_{0}^{+\infty} d r \varphi^{\prime}(r)\left(\frac{1}{r^{n+3}} \int_{\partial B_{r}} v_{\infty}^{2}\right) \geq \int_{0}^{+\infty} d r \varphi(r)\left\{\frac{2}{r} \int_{B_{r}} \frac{1}{|x|^{n}}\left|\frac{U_{\infty}(x)}{|x|^{2}}\right|^{2}\right\}
$$

where $U_{\infty}=x \cdot \nabla v_{\infty}-2 v_{\infty}$. This implies in particular that

$$
\frac{d}{d r}\left(\frac{1}{r^{n+3}} \int_{\partial B_{r}} v_{\infty}^{2}\right) \geq \frac{2}{r} \int_{B_{r}} \frac{1}{|x|^{n}}\left|\frac{U_{\infty}(x)}{|x|^{2}}\right|^{2} \quad \text { in } \quad \mathcal{D}^{\prime}(0,+\infty)
$$

and for every $\lambda>1$ and $s>0$

$$
\left(\frac{1}{(\lambda s)^{n+3}} \int_{\partial B_{\lambda s}} v_{\infty}^{2}\right) \geq\left(\frac{1}{s^{n+3}} \int_{\partial B_{s}} v_{\infty}^{2}\right)+\int_{s}^{\lambda s} d r\left\{\frac{2}{r} \int_{B_{r}} \frac{1}{|x|^{n}}\left|\frac{U_{\infty}(x)}{|x|^{2}}\right|^{2}\right\}
$$

Similarly, for any $q \in \bar{\partial}_{P_{\infty}} \mathcal{P}_{\text {sing }}$, we can do the same reasoning with $v_{m}$ replaced by $v_{m}-q_{m}$ with $q_{m}=\frac{\bar{P}_{m}-P_{m}}{\varepsilon_{m}} \longrightarrow q$ for some $\bar{P}_{m} \in \mathcal{P}_{\text {sing }}$ which gives $(7.82)$ with $v_{\infty}$ replaced by $v_{\infty}-q$, namely

$$
\left(\frac{1}{(\lambda s)^{n+3}} \int_{\partial B_{\lambda s}}\left(v_{\infty}-q\right)^{2}\right) \geq\left(\frac{1}{s^{n+3}} \int_{\partial B_{s}}\left(v_{\infty}-q\right)^{2}\right)+\int_{s}^{\lambda s} d r\left\{\frac{2}{r} \int_{B_{r}} \frac{1}{|x|^{n}}\left|\frac{U_{\infty}(x)}{|x|^{2}}\right|^{2}\right\}
$$

We now remark that for $w_{q}=v_{\infty}-q$, we have

$$
\Gamma_{q}(\rho):=\frac{1}{\rho^{n+4}} \int_{B_{\rho}} w_{q}^{2}=\frac{1}{\rho^{n+4}} \int_{0}^{\rho} d s s^{n+3} \gamma_{q}(s) \quad \text { with } \quad \gamma_{q}(s):=\left(\frac{1}{s^{n+3}} \int_{\partial B_{s}} w_{q}^{2}\right)
$$


Then by integration of $(7.83)$ for $s \in(0, \rho)$, we get

$$
\Gamma_{q}(\lambda \rho) \geq \Gamma_{q}(\rho)+\frac{1}{\rho^{n+4}} \int_{0}^{\rho} d s s^{n+3}\left(\int_{s}^{\lambda s} d r\left\{\frac{2}{r} \int_{B_{r}} \frac{1}{|x|^{n}}\left|\frac{U_{\infty}(x)}{|x|^{2}}\right|^{2}\right\}\right)
$$

Taking the infimum for $q \in \bar{\partial}_{P_{\infty}} \mathcal{P}_{\text {sing }}$ for $\rho=1$, we get

$$
\begin{aligned}
& \inf _{q \in \bar{\partial}_{P_{\infty} \mathcal{P}_{\text {sing }}}}\left(\frac{1}{\lambda^{n+2 p}} \int_{B_{\lambda}}\left|v_{\infty}-q\right|^{2}\right) \\
& \geq \inf _{q \in \bar{\partial}_{P_{\infty}} \mathcal{P}_{\text {sing }}}\left(\int_{B_{1}}\left|v_{\infty}-q\right|^{2}\right)+\int_{0}^{1} d s s^{n+3}\left(\int_{s}^{\lambda s} d r\left\{\frac{2}{r} \int_{B_{r}} \frac{1}{|x|^{n}}\left|\frac{U_{\infty}(x)}{|x|^{2}}\right|^{2}\right\}\right)
\end{aligned}
$$

Using (7.77) and (7.78), we conclude that the second term of the last line is zero for any $\lambda>1$, and then $U_{\infty}=0$, i.e. $v_{\infty}$ is homogeneous of degree 2 .

Therefore, by Lemma 7.6, and the fact that $v_{\infty}$ is superharmonic (see (7.76)), we deduce that $v_{\infty}$ is harmonic on the whole space.

\section{Step 5: Properties of the minimizer $P_{m}$ and consequences}

We write $P_{\infty}(x)=\frac{1}{2}^{t} x \cdot Q_{\infty} \cdot x$ with a diagonal matrix $Q_{\infty}$ (up to rotations and symmetry of the coordinates)

$$
Q_{\infty}=\left(\begin{array}{lllll}
a_{\infty}^{1} & 0 & 0 & \ldots & 0 \\
0 & a_{\infty}^{2} & 0 & \ldots & 0 \\
\ldots & \ldots & \ldots & \ldots & \ldots \\
0 & 0 & 0 & \ldots & a_{\infty}^{n}
\end{array}\right)
$$

with $a_{\infty}^{1} \geq a_{\infty}^{2} \geq \ldots \geq a_{\infty}^{n} \geq 0$. We also recall that trace $Q_{\infty}=1$. Similarly ( $\operatorname{still}$ up to rotations and symmetry of the coordinates), we can assume that we can write $P_{m}(x)=$ ${ }_{2}{ }^{t} x \cdot Q_{m} \cdot x$ with a diagonal matrix $Q_{m}$ (up to rotations)

$$
Q_{m}=\left(\begin{array}{lllll}
a_{m}^{1} & 0 & 0 & \ldots & 0 \\
0 & a_{m}^{2} & 0 & \ldots & 0 \\
\ldots & \ldots & \ldots & \ldots & \ldots \\
0 & 0 & 0 & \ldots & a_{m}^{n}
\end{array}\right)
$$

with $a_{m}^{1} \geq a_{m}^{2} \geq \ldots \geq a_{m}^{n} \geq 0$, trace $Q_{m}=1$ and

$$
a_{m}^{i} \longrightarrow a_{\infty}^{i} \quad \text { for } \quad i=1, \ldots, n
$$

Let us call $k_{\infty}$ the rank of the matrix $Q_{\infty}$, and $k_{m}$ the rank of the matrix $Q_{m}$ which then satisfies $k_{m} \geq k_{\infty}$ (for $m$ large enough).

The fact that $a_{m}^{i}=0$ for $i=k_{m}+1, \ldots, n$, joint to the non-negativity of $u_{m}$, then implies that

$$
v_{m} \geq 0 \quad \text { on } \quad\left\{x_{i}=0, \quad i=1, \ldots, k_{m}\right\}
$$

By hypothesis the energy for $q \in \varepsilon_{m}^{-1}\left(\mathcal{P}_{\text {sing }}-P_{m}\right)$

$$
\mathcal{E}(q):=\int_{B_{1}}\left(v_{m}-q\right)^{2}
$$


is minimal for $q=0$.

Because we have $a_{m}^{i}>0$ for $i=1, \ldots, k_{m}$, we see that the first variation of this energy with respect to variations of the whole coefficients (constraint to stay non-negative with $\left.a_{m}^{1}+\ldots+a_{m}^{n}=1\right)$, implies that

$$
\int_{B_{1}} v_{m}\left(\sum_{i=1}^{n} \gamma_{i} x_{i}^{2}\right) \leq 0 \text { for every }\left\{\begin{array}{l}
\gamma=\left(\gamma_{1}, \ldots, \gamma_{n}\right) \in \mathbb{R}^{k_{m}} \times[0,+\infty)^{n-k_{m}} \\
\text { with } \sum_{i=1}^{n} \gamma_{i}=0
\end{array}\right.
$$

Let us also remark that defining for $i<j$

$$
R_{\theta}(x)=\left(x_{1}, \ldots, x_{i-1}, x_{i} \cos \theta+x_{j} \sin \theta, x_{i+1}, \ldots, x_{j-1}, x_{j} \cos \theta-x_{i} \sin \theta, x_{j+1}, \ldots, x_{n}\right)
$$

and

$$
q_{\theta}(x)=P_{m}\left(R_{\theta}(x)\right)-P_{m}(x)
$$

and derivating the energy $\mathcal{E}\left(q_{\theta}\right)$ with respect to $\theta$ in $\theta=0$, we get

$$
\int_{B_{1}} v_{m}\left(a_{m}^{i}-a_{m}^{j}\right) x_{i} x_{j}=0 \quad \text { for } \quad i, j=1, \ldots, n
$$

Because $a_{m}^{i}>0$ for $i=1, \ldots, k_{m}$, we see that $q_{\eta}(x)=\eta x_{i} x_{j}$ for $i, j \in\left\{1, \ldots, k_{m}\right\}$ with $i \neq j$, satisfies $q_{\eta} \in \varepsilon_{m}^{-1}\left(\mathcal{P}_{\text {sing }}-P_{m}\right)$ for $\eta$ small enough. The first variation of the energy with respect to $\eta$ in $\eta=0$ gives

$$
\int_{B_{1}} v_{m} x_{i} x_{j}=0 \quad \text { for } \quad i, j=1, \ldots, k_{m}, \quad i \neq j
$$

From equations (7.84)-(7.85), we deduce in particular that

$$
\int_{B_{1}} v_{m} x_{i} x_{j}=0 \quad \text { for } \quad i=1, \ldots, k_{m}, \quad j=1, \ldots, n, \quad j \neq i
$$

Let us call $\bar{k}_{\infty}$ the limit of the $k_{m}$ (which satisfies $\bar{k}_{\infty} \geq k_{\infty}$ ). Passing to the limit, we get that $v_{\infty}$ satisfies the assumptions of Lemma 7.7. We deduce that $v_{\infty}=0$. Contradiction with (7.72).

This ends the proof of the Proposition.

\section{Applications}

We now consider obstacle problems for more general elliptic operators. Given an open set $\Omega \in \mathbb{R}^{n}$, we consider solutions $u \in H^{2}(\Omega)$ to the following obstacle problem

$$
\left\{\begin{array}{l|l}
\sum_{i, j=1}^{n} a_{i j}(x) u_{i j}+\sum_{i}^{n} b_{i}(x) u_{i}+c(x) u=f(x) \cdot 1_{\{u>0\}} & \text { on } \Omega \\
u \geq 0 &
\end{array}\right.
$$


where $u_{i j}$ and $u_{i}$ stand respectively for $\frac{\partial^{2} u}{\partial x_{i} \partial x_{j}}$ and $\frac{\partial u}{\partial x_{i}}$. We assume that the coefficients $a_{i j}, b_{i}, c, f$ are continuous and satisfy the following ellipticity/nondegeneracy condition

$$
\exists \delta_{1}>0, \quad \forall x \in \Omega, \quad \forall \xi \in \mathbb{R}^{n}, \quad \sum_{i, j=1}^{n} a_{i j}(x) \xi_{i} \xi_{j} \geq \delta_{1}|\xi|^{2} \quad \text { and } \quad f(x) \geq \delta_{1}
$$

\subsection{Reduction of the problem}

Let us fix a point $x_{0} \in \Omega \cap \partial\{u>0\}$. Then, assuming that the coefficients $a_{i j}, b_{i}, c, f$ are Dini, we can rewrite the first line of (8.87) as

$$
\sum_{i, j=1}^{n} a_{i j}\left(x_{0}\right) u_{i j}=\tilde{f}_{x_{0}}(x) \cdot 1_{\{u>0\}}
$$

where the function $\tilde{f}_{x_{0}}$ is defined by

$$
\tilde{f}_{x_{0}}(x)=f(x)-\sum_{i, j=1}^{n}\left(a_{i j}(x)-a_{i j}\left(x_{0}\right)\right) u_{i j}-\sum_{i}^{n}\left(b_{i}(x)-b_{i}\left(x_{0}\right)\right) u_{i}-\left(c(x)-c\left(x_{0}\right)\right) u
$$

Then up to diagonalize the matrix $\left(a_{i j}\left(x_{0}\right)\right)_{i j}$ and to change the coordinates, we see that there exists a matrix $A_{x_{0}}$ such that if we define $u_{x_{0}}(x)=u\left(x_{0}+A_{x_{0}} \cdot x\right), f_{x_{0}}(x)=\tilde{f}_{x_{0}}\left(x_{0}+A_{x_{0}} \cdot x\right)$, then we have

$$
\left\{\begin{array}{l}
\Delta u_{x_{0}}=f_{x_{0}}(x) \cdot 1_{\left\{u_{x_{0}}>0\right\}} \\
u_{x_{0}} \geq 0 \\
\frac{1}{\delta_{0}} \geq f_{x_{0}} \geq \delta_{0} \\
f_{x_{0}}(0)=1 \\
0 \in \partial\left\{u_{x_{0}}>0\right\}
\end{array} \mid \text { on } B_{1}\right.
$$

Then we define

$$
\sigma_{x_{0}}(\rho)=\sup _{r \in(0, \rho]}\left(\frac{1}{\left|B_{r}\right|} \int_{B_{r}}\left|f_{x_{0}}(x)-f_{x_{0}}(0)\right|^{p}\right)^{\frac{1}{p}}
$$

In particular we get that $\sigma_{x_{0}}$ is Dini.

Indeed, it is possible to apply the approach of Alt, Phillips [2], which shows that the second derivatives of $u$ are bounded. Their method is based on the interior Schauder estimate, which has to be replaced by a similar estimate for Dini coefficients. This last estimate is for instance a consequence of the estimate of Theorem 2.1, using the classical perturbation method.

Remark 8.1 It would be interesting to provide a pointwise proof that $\sigma_{x_{0}}$ is Dini, avoiding the argument of Alt, Phillips. 


\subsection{Regularity of the free boundary}

We also introduce the following

\section{Definition 8.2 (Dini continuity)}

We say that a function $f$ is Dini continuous, if its modulus of continuity given by (1.2) is Dini in the sense of Definition 1.1.

Then we have the following result

\section{Theorem 8.3 (Regularity of the free boundary)}

Under assumption (8.88), let us consider a solution $u$ of (8.87) with Dini continuous coefficients $a_{i j}, b_{i}, c, f$. Then we can write the free boundary as follows

$$
(\partial\{u>0\}) \cap \Omega=\mathcal{R} \cup \mathcal{S}
$$

i) where $\mathcal{R}$ is locally a $C^{1}$ hypersurface,

ii) and the singular set $\mathcal{S}$ of the free boundary can be written

$$
\mathcal{S}=\bigcup_{j=0}^{n-1} \mathcal{S}_{j}
$$

where each $\mathcal{S}_{j}$ is locally contained in a $C^{1} j$-dimensional manifold.

Remark 8.4 Here the sets $\mathcal{R}(r e s p . \mathcal{S})$ stands for the set of regular points defined via Proposition 1.6 applied to the reduced problem (8.89).

The first claim of this Theorem on the regular part of the free boundary was already proved (with a different proof for the Laplace operator) in Blank [5].

The second claim of the Theorem on the singular part of the free boundary was proved by Caffarelli [8] for Lipschitz coefficients, and by Monneau [32] for Hölder coefficients, including extensions for double Dini coeffifients).

\section{Sketch of the proof of Theorem 8.3}

We already know the uniqueness of the blow-up limit at every regular and singular points. Moreover these limits enjoy a stability property for neighbouring points (using respectively (2.14) and (7.58)). Consequently the map which associate its blow-up limit to each regular point is continuous. This is also the case for singular points. A further inspection of the structure of the blow-up limits $u^{0}(x)=\frac{1}{2}^{t} x \cdot Q \cdot x$ shows that we can consider the singular points $\mathcal{S}_{k}$ associated to a matrix $Q$ of rank $k \leq n-1$.

Finally, the rest of the proof is classical, see for instance Caffarelli [7], [9], Monneau [32] (see also Caffarelli, Shahgholian [16], Caffarelli, Petrosyan, Shahgholian [15], for similar obstacle problem without sign condition on the solution).

This ends the sketch of the proof of the Theorem. 


\section{Appendix : an application to fully nonlinear elliptic equations}

Let us mention that $C^{2, \alpha}$ estimates are known for solutions of concave (or convex) fully nonlinear uniformly elliptic equations (see chapter 6 of Caffarelli, Cabre [11] or its generalizations in chapter 8, see also the original paper of Caffarelli [10]). For results with Dini conditions, see Kovats [28, 29], Zou, Chen [40], and Bao [3], and for the connection between the $C^{2, \alpha}$ regularity of $C^{1,1}$ solutions with the Liouville property, see Huang [26]. Here we show, without assuming concavity or convexity of the equation, a pointwise $C^{2, \alpha}$ estimate (in the $L^{p}$ norm) assuming a pointwise $C^{2}$-Dini regularity (in the $L^{\infty}$ norm).

Proposition 9.1 ( $C^{2}$-Dini implies $C^{2, \alpha}$ for fully nonlinear equations)

Let us consider a function $u \in C^{2}\left(B_{1}\right)$ solution of

$$
F\left(D^{2} u\right)=0 \quad \text { on } \quad B_{1}
$$

with $F \in C^{2}$ and uniformly elliptic.

Let us define for $p \in(1,+\infty)$ (and for $P_{0}$ a polynomial of degree less or equal to 2 )

$$
\omega_{p}(r)=\sup _{\rho \in(0, r]}\left(\frac{1}{\left|B_{\rho}\right|} \int_{B_{\rho}}\left|\frac{u-P_{0}}{\rho^{2}}\right|^{p}\right)^{\frac{1}{p}}
$$

and

$$
\omega_{\infty}(r)=\sup _{\rho \in(0, r]} \sup _{B_{\rho}}\left|\frac{u-P_{0}}{\rho^{2}}\right|
$$

If $\int_{0}^{1} \frac{\omega_{\infty}(r)}{r} d r<+\infty$, then there exist constants $C>0$ and $\alpha, r_{1} \in(0,1]$ such that

$$
\forall r \in\left(0, r_{1}\right), \quad \omega_{p}(r) \leq C r^{\alpha}
$$

\section{Proof of Proposition 9.1}

Up to redefine $u$, we can assume that $D^{2} u(0)=0$ and perform a Taylor expansion of

$$
F\left(D^{2} u\right)=0
$$

as

$$
0=F(0)+F^{\prime}(0) D^{2} u+O\left(\left|D^{2} u\right|^{2}\right)
$$

i.e.

$$
F^{\prime}(0) D^{2} u=O\left(\left|D^{2} u\right|^{2}\right)
$$

We deduce from Theorem 2.1, that for $r<r_{0}$

$$
\begin{aligned}
\left(\frac{1}{\left|B_{r}\right|} \int_{B_{r}}\left|\frac{u(x)-P_{0}(x)}{r^{2}}\right|^{p}\right)^{\frac{1}{p}} & \leq C\left(M r^{\alpha}+\int_{0}^{r} \frac{\omega_{p}(s) \omega_{\infty}(s)}{s} d s+r^{\alpha} \int_{r}^{1} \frac{\omega_{p}(s) \omega_{\infty}(s)}{s^{1+\alpha}} d s\right) \\
& \leq C\left(M r^{\alpha}+\omega_{p}(r) \varepsilon(r)\right)
\end{aligned}
$$

with

$$
\varepsilon(r)=\int_{0}^{r} \frac{\omega_{\infty}(s)}{s} d s+r^{\alpha} \int_{r}^{1} \frac{\omega_{\infty}(s)}{s^{1+\alpha}}
$$


Therefore

$$
\omega_{p}(r) \leq C\left(M r^{\alpha}+\omega_{p}(r) \varepsilon(r)\right)
$$

which implies the result for $r$ small enough. This ends the proof of the Proposition.

\section{Aknowledgements}

I would like to thank Luis Caffarelli and Henrik Shahgholian for indications about the references and stimulating discussions. This work was supported by the ACI "EDP et finance" (2003-2007).

\section{References}

[1] H.W. Alt, L.A. Caffarelli, A. Friedman, Variational Problems with two phases and their free boundaries, Trans. Amer. Math. Soc. 282 (2), (1984), 431-461.

[2] H.W. Alt, D. Phillips, A free boundary problem for semilinear elliptic equations, J. Reine Angew. Math. 368 (1986), 63-107.

[3] J. BAO, Fully Nonlinear Elliptic Equations on General Domains, Canad. J. Math. 54 (6), 1121-1141, (2002).

[4] C. Burch, The Dini Condition and Regularity of Weak Solutions of Elliptic Equations, J. Diff. Eq. 30 (1978), 308-323.

[5] I. Blank, Sharp Results for the Regularity and Stability of the Free Boundary in the Obstacle Problem, Indiana Univ. Math. J., 50(3), 1077-1112, (2002).

[6] L.A. Caffarelli, Free boundary problem in highter dimensions, Acta Math. 139, (1977), 155-184.

[7] L.A. Caffarelli, Compactness Methods in Free Boundary Problems, Comm. Partial Differential Equations 5 (4), (1980), 427-448.

[8] L.A. Caffarelli, The Obstacle Problem revisited, J. Fourier Anal. Appl. 4, (1998), 383-402.

[9] L.A. Caffarelli, The Obstacle Problem, Lezioni Fermiane. Accademia Nazionale dei Lincei, Rome; Scuola Normale Superiore, Pisa, (1998).

[10] L.A. CAFFarelli, Interior a priori Estimates for Solutions of Fully Nonlinear Equations, Annals of Mathematics 130 (1989), 189-213.

[11] L.A. Caffarelli, X. Cabre, Fully Nonlinear Elliptic Equations, Amer. Math. Soc., Providence, R.I., 1995.

[12] L.A. Caffarelli, Q. Huang, Estimates in the generalized Campanato-JohnNirenberg spaces for fully nonlinear elliptic equations, Duke Math. J. 118 (1) (2003), $1-17$.

[13] L.A. Caffarelli, L. Karp, H. Shahgholian, Regularity of a free boundary with application to the Pompeiu problem, Ann. Math. (2) 151 (2000), pp. 269-292. 
[14] L.A. Caffarelli, D. Kinderlehrer, Potential methods in variational inequalities, J. Analyse Math. 37 (1980), 285-295.

[15] L.A. Caffarelli, A. Petrosyan, H. Shahgholian, Regularity of a free boundary in parabolic potential theory, J. Amer. Math. Soc. 17 (4), 827-869, (2004).

[16] L.A. Caffarelli, H. Shahgholian, The structure of the singular set of the free boundary in potential theory, J. Contemp. Math. Anal. 39 (2), 2-30, (2005).

[17] A.P. Calderon, A. Zygmund, Local properties of solutions of elliptic partial differential equations, Studia Math. 20 (1961), 171-225.

[18] S. Campanato, Proprieta di una Famiglia di Spazi Functionali, Ann. Scuola Norm. Sup. Pisa (3) 18 (1964), 137-160.

[19] J. Diederich, Removable Sets for Pointwise Solutions of Elliptic partial Differential Equations, Trans. Amer. Math. Soc. 165 (1972), 333-352.

[20] F. DuzaAR, A. Gastel Nonlinear elliptic systems with Dini continuous coefficients, Arch. Math. 78 (2002), 58-73.

[21] F. Duzaar, A. Gastel, G. Mingione, Elliptic systems, singular sets and Dini continuity, Comm. Partial Differential Equations 29 (2004), No 7-8, 1215-1240.

[22] L.C. Evans, Quasiconvexity and partial regularity in the calculus of variations, Arch. Rational Mech. Anal. 95 (1986), 227-252.

[23] L.C. Evans, R.F. Gariepy, Compactness and Partial Regularity in the Calculus of Variations, Indiana Univ. Math. J. 36 (2) (1987), 361-371.

[24] H. Federer, Geometric Measure Theory, Springer-Verlag, (1969).

[25] D.N. Gilbarg, N.S. Trudinger, Elliptic Partial Differential Equations of Second Order, Springer-Verlag, 3nd edition, (1997).

[26] Q. HuAng, On the regularity of solutions to fully nonlinear elliptic equations via the Liouville property, Proc. Amer. Math. Soc. 130 (2002), 1955-1959.

[27] P. Hartman, A. Wintner, On Uniform Dini Conditions in the Theory of Linear Partial Differential Equations of Elliptic Type, Amer. J. Math. 77 (1954), 329-354.

[28] J. Kovats, Fully nonlinear elliptic equations and the Dini condition, Comm. PDE 22, 1911-1927 (1997).

[29] J. Kovats, Dini-Campanato spaces and applications to nonlinear elliptic equations, Electronic Journal of Differential Equations 37 (1999), pp. 1-20.

[30] K. Lee, H. Shahgholian Regularity of a free boundary for viscosity solutions of nonlinear elliptic equations, Comm. Pure Appl. Math. 54 (1), 43-56, (2001).

[31] M.I. Matilchuk, S.D. Eidel'man, The Cauchy Problem for Parabolic Systems whose Coefficients Have Slight Smoothness, Ukrainian Math. J. 22 (1970), 18-30. 
[32] R. Monneau, On the number of singularities for the obstacle problem in two dimensions, J. Geom. Anal. 13 (2), 359-389, (2003).

[33] R. Monneau, A Brief Overview on the Obstacle Problem, in Proceedings of the Third European Congress of Mathematics, Barcelona, (2000): Progress in Mathematics, Vol. 202, Birkhäuser Verlag Basel/Switzerland, (2001), 303-312.

[34] A. Petrosyan, H. Shahgholian, Geometric and energetic criteria for the free boundary regularity in an obstacle problem, preprint (2006).

[35] L. Simon, Asymptotics for a class of nonlinear evolution equations, with applications to geometric problems. Ann. of Math. (2) 118, no. 3, 525-571, (1983).

[36] L. Simon, Schauder estimates by scaling. Calc. Var. Partial Differential Equations 5 (5), 391-407, (1997).

[37] L. WAng, On the Regularity of Fully Nonlinear parabolic Equations II, Comm. Pure and Applied Math. 45 (1992), 141-178.

[38] G.S. WeISS, A homogeneity improvement approach to the obstacle problem, Invent. math. 138, (1999), 23-50.

[39] J. Wolf, Partial regularity of weak solutions to nonlinear elliptic systems satisfying a Dini condition, Z. Anal. Anwendungen 20 (2), 315-330, (2001).

[40] X. Zou, Y.Z. Chen, Fully Nonlinear Parabolic Equations and the Dini Condition, Acta Mathematica Sinica, English series, July, 2002, Vol. 18, No. 3, pp. 473-480. 